\title{
Modularity and Dynamic Adaptation of Flexibly Secure Systems: Model-Driven Adaptive Delegation in Access Control Management
}

\author{
Phu H. Nguyen, Gregory Nain, Jacques Klein, \\ Tejeddine Mouelhi, and Yves Le Traon \\ Interdisciplinary Centre for Security, Reliability and Trust (SnT) \\ University of Luxembourg \\ 4 rue Alphonse Weicker, L-2721 Luxembourg \\ \{phuhong.nguyen, gregory.nain, jacques.klein, \\ tejeddine.mouelhi, yves.letraon\}@uni.lu
}

\begin{abstract}
Model-Driven Security (MDs) is a specialized Model-Driven Engineering (MDE) approach for supporting the development of secure systems. Model-Driven Security aims at improving the productivity of the development process and quality of the resulting secure systems, with models as the main artifact. Among the variety of models that have been studied in a Model-Driven Security perspective, one can mention access control models that specify the access rights. So far, these models mainly focus on static definitions of access control policies, without taking into account the more complex, but essential, delegation of rights mechanism. Delegation is a meta-level mechanism for administrating access rights, which allows a user without any specific administrative privileges to delegate his/her access rights to another user. This paper gives a formalization of access control and delegation mechanisms, and analyses the main hard-points for introducing various advanced delegation semantics in Model-Driven Security. Then, we propose a modular model-driven framework for 1) specifying access control, delegation and the business logic as separate concerns; 2) dynamically enforcing/weaving access control policies with various delegation features into securitycritical systems; and 3) providing a flexibly dynamic adaptation strategy. We demonstrate the feasibility and effectiveness of our proposed solution through the proof-of-concept implementations of different componentbased systems running on different adaptive execution platforms, i.e. OSGi and Kevoree.
\end{abstract}

Keywords: Model-driven security, model-driven engineering, MDE, model composition, delegation, access control, dynamic adaptation, OSGi, Kevoree

\section{Introduction}

Software security is a polymorphic concept that encompasses different viewpoints (hacker, security officer, end-user) and raises complex management issues when 
considering the ever increasing complexity and dynamism of modern software. In this perspective, designing, implementing, and testing software for security is a hard task, especially because security is dynamic, meaning that a security policy can be updated at any time and that it must be kept aligned with the software evolution. As one of the key concerns in software security, managing access control to critical resources requires the dynamic enforcement of access control policies. Access control policies stipulate actors access rights to internal resources and ensure that users can only access the resources they are allowed to in a given context. A sound methodology supporting such security-critical systems development is extremely necessary because access control mechanisms cannot be "blindly" inserted into a system, but the overall system development must take access control aspects into account. Critical resources could be accessible to wrong (or even malicious) users just because of a small error in the specification or in the implementation of the access control policy.

Several design approaches like [24] [4] have been proposed to enable the enforcement of classical security models, such as Role-Based Access Control (RBAC) [12] [32]. These approaches bridge the gap from the high-level definition of an access control policy to its enforcement in the running software, automating the dynamic deployment of a given access control policy. Although such a bridge is a prerequisite for the dynamic administration of a given access control policy, it is not sufficient to offer the advanced administration instruments that are necessary to efficiently manage access control. In particular, delegation of rights is a complex dimension of access control that has not yet been addressed by the adaptive access control mechanisms. User delegation is necessary for assigning permissions from one user to another user. An expressive design of access control must extensively take into account delegation requirements.

Delegation models based on RBAC management have been characterized as secure, flexible and efficient access management for resource sharing, especially in a distributed environment. Flexible means that different subjects for delegation should be supported, i.e. delegation of roles, specific permissions or obligations. Also, different features of delegation should be supported, like temporary and recurrent delegation, transfer of role or permissions, delegation to multiple users, multi-step delegation, revocation, etc. However, the addition of flexibility for delegation must come with mechanisms to make sure that the security policy of the system is securely consistent. And last but not least, the administration of delegations must remain simple to be efficient. Thus, delegation is a complex problem to solve and to our best knowledge, there has been no complete approach for both specifying and dynamically enforcing access control policies by taking into account various features of delegation. Having such an expressive security model is crucial in order to simplify the administrative task and to manage collaborative work securely, especially with the increase in shared information and distributed systems.

Based on previous work [24], in this paper we propose a new Modular ModelDriven Security solution to easily and separately specify 1) the business logic of the system without any security concern using a Domain Specific Modeling Lan- 
guage (DSML) for describing the architecture of a system in terms of components and bindings; 2) the "traditional" access control policy using a DsmL based on a RBAC-based metamodel; 3) an advanced delegation policy based on a DsML dedicated to delegation management. In this third DsML, delegation can be seen as a "meta-level" mechanism which impacts the existing access control policies similarly as an aspect can impact a base program. The security enforcement is enabled by leveraging automated model transformation/composition (from security model to architecture model). Consequently, in addition to [24], an advanced model composition is required to correctly handle the new delegation features. In this paper, we claim that delegation needs to be clearly separated from access control because a delegation policy impacts access control rules. Therefore, delegation and access control are not at the same level and should be separated. This separation involves an advanced model composition approach to dynamically know, at any time, what is the set of new access controls that has to be considered, i.e., the "normal" access control rules as well as the access control rules modified by the delegation rules. From a more technical point of view, the security enforcement is dynamically done via automated model transformation/composition (from security model to architecture model) and the dynamic reconfiguration ability of modern adaptive execution platforms.

This paper is an extension of our earlier paper [28] which was presented at the conference Modularity: AOSD'13. The remainder of this paper is organized as follows. Section 2 presents the background on access control, delegation, and the security-driven model-based dynamic adaptation. Formal definitions of our access control model and formalisms of advanced delegation features are given in detail. Section 3 describes a running example. It will be used throughout the paper to show the diverse characteristics of delegation and illustrate the various aspects of our approach. In Section 4, we first give an overview of our approach. Then, we formalize our delegation mechanisms based on RBAC and show how our delegation metamodel can be used to specify expressive access control policies that take into account various features of delegation. Based on the delegation metamodel, we describe our model transformation/composition rules used for transforming and weaving security policy into an architecture model. This section ends with a discussion of several strategies for dynamic adaptation and evolution of security policy. Section 5 describes how our approach has been applied and evaluated in the development of three different systems running on two different adaptive execution platforms. Next, related work is presented in Section 6. Finally, Section 7 concludes the paper and discusses future work.

\section{Background}

This section introduces the main concepts which are used in this paper. Firstly, formal definitions of Access Control and Delegation policies are presented. Based on these definitions, some key advanced delegation features are introduced formally. We keep all the definitions here generic so that they can be mapped into different security models like Role-Based Access Control (RBAC), Organization- 
Based Access Control (Orbac) [16], Discretionary Access Control (DAC) [19], etc. These definitions also provide the basis for deriving mutation operators that can be used for testing delegation policy enforcement [29]. Then, a brief summary of previous work on dynamic security policy enforcement [24] is given.

\subsection{Access Control}

Access Control [14] is known as one of the most important security mechanisms. It enables the regulation of user access to system resources by enforcing access control policies. A policy defines a set of access control rules which expresses: who has the right to access a given resource or not, and the way to access it, i.e. which actions a user can access under which conditions or contexts.

Definition 1 (Access Control). Let $U$ be a set of users, $P$ be a set of permissions, and $C$ be a set of contexts. An access control policy $A C$ is defined as a user-permission-context assignment relation: $A C \subseteq U \times P \times C$. A user $u$ is granted permission $p$ in a given context $c$ if and only if $(u, p, c) \in A C$.

Additional details about contexts are given in next Sub-Section 2.2.

\subsection{Delegation}

In the field of access control, delegation is a very complex but important aspect that plays a key role in the administration mechanism [5]. A software system which supports delegation, should allow its users without any specific administrative privileges to grant some authorizations. Delegation of rights allows a user, called the delegator, to delegate his/her access rights to another user, called the delegatee. By this delegation, the delegatee is allowed to perform the delegated roles/permissions on behalf of the delegator [9]. The delegator has full responsibility and accountability for the delegated accesses since he/she provides the accesses to the resources to other users, who are not initially authorized by the access control rules to access these resources.

A delegation policy can be considered as an administration-related security policy that is built on top of an access control policy. It is composed of delegation rules that can be specified at two levels: master-level and user-level. Basically, a delegation policy is two-fold:

1. It specifies who has the right to delegate which permission (for accessing to a given resource/action/subject) to whom, and in which context. We call this kind of rule master-level delegation rule as such a rule is normally defined by security officers. For example, a security officer can define a rule to specify that the head of a department at a university can only delegate the permission of updating personnel accounts to a professor.

Definition 2 (Master-Level Delegation Policy). Let $U$ be a set of users, $P$ be a set of permissions, and $C$ be a set of contexts. A master-level delegation policy MLD is defined as a user-user-permission-context assignment relation: 
$M L D \subseteq U \times U \times P \times C$ with the following meaning. A delegation of a permission $p$ from a user $u_{1}$ to a user $u_{2}$ in a given context $c$ is allowed if and only if $\left(u_{1}, u_{2}, p, c\right) \in M L D$.

2. It specifies who delegates to whom which permission, and in which context. We call this kind of rule user-level delegation rule as these rules are mostly defined by normal users. Note that user-level delegation rules must conform to master-level delegation rules. For example, Bill (the head of department) delegates his permission of updating personnel accounts to Bob (a professor) during his absence.

Definition 3 (User-Level Delegation Policy). Let $U$ be a set of users, $P$ be a set of permissions, and $C$ be a set of contexts. A user-level delegation policy $U L D$ is defined as a user-user-permission-context assignment relation: $U L D \subseteq$ $U \times U \times P \times C$ with the following meaning. A user $u_{2}$ has a permission $p$ by delegation from a user $u_{1}$ in a given context $c$ if and only if $\left(u_{1}, u_{2}, p, c\right) \in U L D$. It can be seen that all the delegations in $U L D$ conform to the rules defined in the $M L D$. In other words, every delegation at the user-level can only be created if it conforms to the delegation rules defined at the master-level.

Context A context is a condition or a combination of conditions in which an access control/delegation rule is active, i.e. enforced in the running system. Cuppens et al. discuss five different kinds of contexts in [10]. These kinds of contexts include temporal context, spatial context, user-declared context, prerequisite context, and provisional context. Temporal delegation is delegation within a time constraint, for example delegation is active for two days, or delegation is active for the time the delegator is on vacation. The spatial context relies on the delegator/delegatee's location, e.g. a delegated permission is only active when the delegatee is at office. User-declared context is related to the purpose of the delegator/delegatee, e.g. a delegator may state that his/her delegatee cannot further delegate his/her permissions to someone else. Prerequisite context allows delegation when some precondition is satisfied and the provisional context depends on the previous actions that delegator/delegatee has performed on the system. Moreover, it is possible for a security rule to have a complex context, which is a composition of contexts. Our security model supports context composition using conjunction \&, disjunction $\oplus$, and negation ${ }^{-}$.

Note that every access control rule and delegation rule defined in this paper is always associated with a context $c$. By default, if not specified explicitly, a context $c$ is at least composed of a condition, called Default, i.e. always true.

\subsection{Advanced Delegation Features}

Delegation is a powerful and very useful way to augment access control policy administration. On one hand, it allows users to temporarily modify the access control policy by delegating access rights. By delegation, a delegatee can perform the delegated job, without requiring the intervention of the security officer. 
On the other hand, the delegator and/or some specific authorized users should be supported to revoke the delegation either manually or automatically. In both cases, the administrative task can be simplified and collaborative work can be managed securely, especially with the increase in shared information and distributed systems [1]. However, the simpler the administrative task can be, the more complex features of delegation have to be properly specified and enforced in the software system. To the best of our knowledge, there is no approach for both specifying and dynamically enforcing access control policies taking into account all delegation features like temporary delegation, transfer delegation, multiple delegation, multi-step delegation, etc.

In this section, we define the most well-known complex delegation features and formally specify them w.r.t. the definitions of access control and delegation policies. In the following definitions, we use pre, body, and post to respectively specify the state of the policy before changing, the state while it is being changed by the function (the delegation rule is being enforced), and the state after changing.

Monotonicity of Delegation Monotonicity of delegation refers to whether or not the delegator can still use the permission while delegating it [9]. If the delegator can still use the permission while delegating it, the delegation is called grant delegation. Of course, the delegatee can use the permission while it is delegated to him. This is monotonic because available authorizations (in the set $A C$ ) are increased due to successful delegation operations. Again, note that every delegation can only be performed if and only if it satisfies the master-level delegation policy.

Definition 4 (Grant Delegation). grantDelegation $\left(u_{1}, u_{2}, p, c\right)$ : pre $\left(u_{1}, p, c\right) \in A C \wedge\left(u_{2}, p, c\right) \notin A C \wedge\left(u_{1}, u_{2}, p, c\right) \in M L D$ body $A C:=A C \cup\left\{\left(u_{2}, p, c\right)\right\} ; U L D:=U L D \cup\left\{\left(u_{1}, u_{2}, p, c\right)\right\}$ end post $\left(u_{1}, p, c\right) \in A C \wedge\left(u_{2}, p, c\right) \in A C \wedge\left(u_{1}, u_{2}, p, c\right) \in U L D$

Vice versa, if the delegator can not use the permission while delegating it, the delegation is called transfer delegation. As such, this is non-monotonic because available authorizations (in $A C$ ) are not increased due to successful delegation operations.

Definition 5 (Transfer Delegation). transferDelegation $\left(u_{1}, u_{2}, p, c\right)$ : pre $\left(u_{1}, p, c\right) \in A C \wedge\left(u_{2}, p, c\right) \notin A C \wedge\left(u_{1}, u_{2}, p, c\right) \in M L D$ body $A C:=A C \backslash\left\{\left(u_{1}, p, c\right)\right\} ; A C:=A C \cup\left\{\left(u_{2}, p, c\right)\right\} ; U L D:=U L D \cup$ $\left\{\left(u_{1}, u_{2}, p, c\right)\right\}$ end

post $\left(u_{1}, p, c\right) \notin A C \wedge\left(u_{2}, p, c\right) \in A C \wedge\left(u_{1}, u_{2}, p, c\right) \in U L D$

Temporary Delegation This is also a very common feature of delegation needed by users. When revocation is handled automatically, the delegation is called temporary. In this case, the delegator specifies the temporal conditions in which this delegation applies: only at a given time, after or before a given time, 
or during a given time interval. The temporal conditions may correspond to a day of the week, or to a time of the day, etc. If the temporal context is not used, the delegation needs to be revoked manually.

Definition 6 (Temporary Delegation). Let $c$ be a given context of a delegation (either grant delegation or transfer delegation). A delegation is specified as temporary if its context $c$ is associated with a time constraint. The delegation will only be active while the time constraint is satisfied.

For example, if the context is vacation period, a delegator Bill could have an associated delegation rule with the following context:

$c:=$ c\&vacation_period(startDate, endDate)

where vacation_period(startDate, endDate) :-

startDate $\leq$ endDate $\wedge$ afterDate $($ startDate $) \wedge$ be foreDate (endDate $)$

Here, afterDate(date) returns true iff date is equal or later than the current date. Similarly, beforeDate(date) returns true iff date is equal or earlier than the current date.

Multiple Delegation A permission can be delegated to more than a delegatee at a given time. However, the number of times that a permission is concurrently delegated have to be controlled. Multiple delegation refers to the maximum number of times that a permission can be delegated at a given time.

Definition 7 (Multiple Delegation). Let $N_{m}$ be the maximum number of times that a permission can be concurrently delegated. $N_{m}$ is predefined by the security officer. The number of concurrent delegations in which the same role or permission is delegated at a given time, in a given context can not exceed $N_{m}$.

We introduce a counting function to count the number of delegations of a permission which is delegated by a delegator in a given context. The number returned by this function is always updated according to the change in the delegation policy, i.e. the number of delegation rules related to permission $p$.

countDelegation $(u, p, c):=|\{(u, v, p, c) \mid \forall v \in U:(u, v, p, c) \in U L D\}|$

If the number of concurrent delegations of the same permission at a given time, in a given context has not exceeded $N_{m}$, then this permission is still allowed to be delegated.

grantDelegation $\left(u_{1}, u_{2}, p\right.$

c\&countDelegation $\left.\left(u_{1}, p, c\right)<N_{m}\right):-\operatorname{pre}\left(u_{1}, p, c\right) \in A C \wedge\left(u_{2}, p, c\right) \notin A C \wedge$

$\left(u_{1}, u_{2}, p, c\right) \in M L D \wedge$ countDelegation $\left(u_{1}, p, c\right)<N_{m}$

body $A C:=A C \cup\left\{\left(u_{2}, p, c\right)\right\} ; U L D:=U L D \cup\left\{\left(u_{1}, u_{2}, p, c\right)\right\}$ end

post $\left(u_{1}, p, c\right) \in A C \wedge\left(u_{2}, p, c\right) \in A C \wedge\left(u_{1}, u_{2}, p, c\right) \in U L D$

Multi-step Delegation This characteristic refers to the maximum number of steps $\left(N_{s}\right.$, normally specified by a security officer) that a permission $p$ can be re-delegated, counted from the first delegator of this permission. So if $N_{s}=0$ that means the permission $p$ can not be re-delegated anymore. 
Definition 8 (Multi-step Delegation). Let $N_{s} \geq 0$ be the maximum number of steps that a permission $p$ can be re-delegated. A permission $p$ can only be delegated iff $N_{s}>0$.

First, let us define a helper function that returns the number of times a permission $p$ is re-delegated in a given context c. stepCounter $\left(u_{0}, p, c\right):=N_{s}$ where $u_{0}$ is the first delegator of $p$ in the delegation chain: $u_{0}$ delegates $p$ to $\ldots$ in a given context $c ; \ldots$ re-delegates $p$ to $u_{1}$ in context $c$; and $u_{1}$ re-delegates $p$ to $u_{2}$ in context $c$. Here, "...." is the users in the middle of the delegation chain, $u_{1}$ is the current last delegatee of this chain, and $u_{2}$ is the next delegatee if stepCounter $\left(u_{1}, p, c\right) \geq 1$.

If there exists a predefined maximum number of steps $N_{s}$ for a permission $p$ as described above, the delegation is specified as following.

grantDelegation $\left(u_{1}, u_{2}, p, c\right.$ \&stepCounter $\left.\left(u_{1}, p, c\right) \geq 1\right):-$ pre $\left(u_{1}, p, c\right) \in A C \wedge\left(u_{2}, p, c\right) \notin A C \wedge\left(u_{1}, u_{2}, p, c\right) \in M L D \wedge \operatorname{stepCounter}\left(u_{1}, p, c\right) \geq$ 1

body $A C:=A C \cup\left\{\left(u_{2}, p, c\right)\right\} ; U L D:=U L D \cup\left\{\left(u_{1}, u_{2}, p, c\right)\right\} ;$ stepCounter $\left(u_{2}, p, c\right):=$ stepCounter $\left(u_{1}, p, c\right)-1$ end

post $\left(u_{1}, p, c\right) \in A C \wedge\left(u_{2}, p, c\right) \in A C \wedge\left(u_{1}, u_{2}, p, c\right) \in U L D$

Delegation Revocation Delegation supports a revocation feature in which a delegation can be revoked and permissions are returned back to the original user.

Definition 9 (Delegation Revocation). Delegation revocation is the ability for any delegation can be manually revoked by authorized users.

The revocation of a grant delegation means to deny access of the delegatee to the delegated permission.

Definition 10. revokeGrantDelegation $\left(u_{1}, u_{2}, p, c\right):-$ pre $\left(u_{1}, p, c\right) \in A C \wedge\left(u_{2}, p, c\right) \in A C \wedge\left(u_{1}, u_{2}, p, c\right) \in U L D$ body $A C:=A C \backslash\left\{\left(u_{2}, p, c\right)\right\} ; U L D:=U L D \backslash\left\{\left(u_{1}, u_{2}, p, c\right)\right\}$ end post $\left(u_{1}, p, c\right) \in A C \wedge\left(u_{2}, p, c\right) \notin A C \wedge\left(u_{1}, u_{2}, p, c\right) \notin U L D$

The permission to be revoked is deleted from the access rights of the delegatee. To revoke a transfer delegation, it is not only to deny access of the delegatee to the delegated permission but also to re-grant access to the delegator who is temporarily not having this access.

Definition 11. revokeTransferDelegation $\left(u_{1}, u_{2}, p, c\right):-$ pre $\left(u_{2}, p, c\right) \in A C \wedge\left(u_{1}, p, c\right) \notin A C \wedge\left(u_{1}, u_{2}, p, c\right) \in U L D$ body $A C:=A C \backslash\left\{\left(u_{2}, p, c\right)\right\} ; A C:=A C \cup\left\{\left(u_{1}, p, c\right)\right\} ; U L D:=U L D \backslash\left\{\left(u_{1}, u_{2}, p, c\right)\right\}$ end post $\left(u_{1}, p, c\right) \in A C \wedge\left(u_{2}, p, c\right) \notin A C \wedge\left(u_{1}, u_{2}, p, c\right) \notin U L D$ 
We have presented formal definitions of access control, delegation, and various delegation features. These definitions are generic (at the conceptual level) so that they can be mapped into different security models like RBAC, ORBAC, DAC, etc. Section 4 shows how these formal concepts can be implemented (based on RBAC) using MDE techniques.

\subsection{Security-Driven Model-Based Dynamic Adaptation}

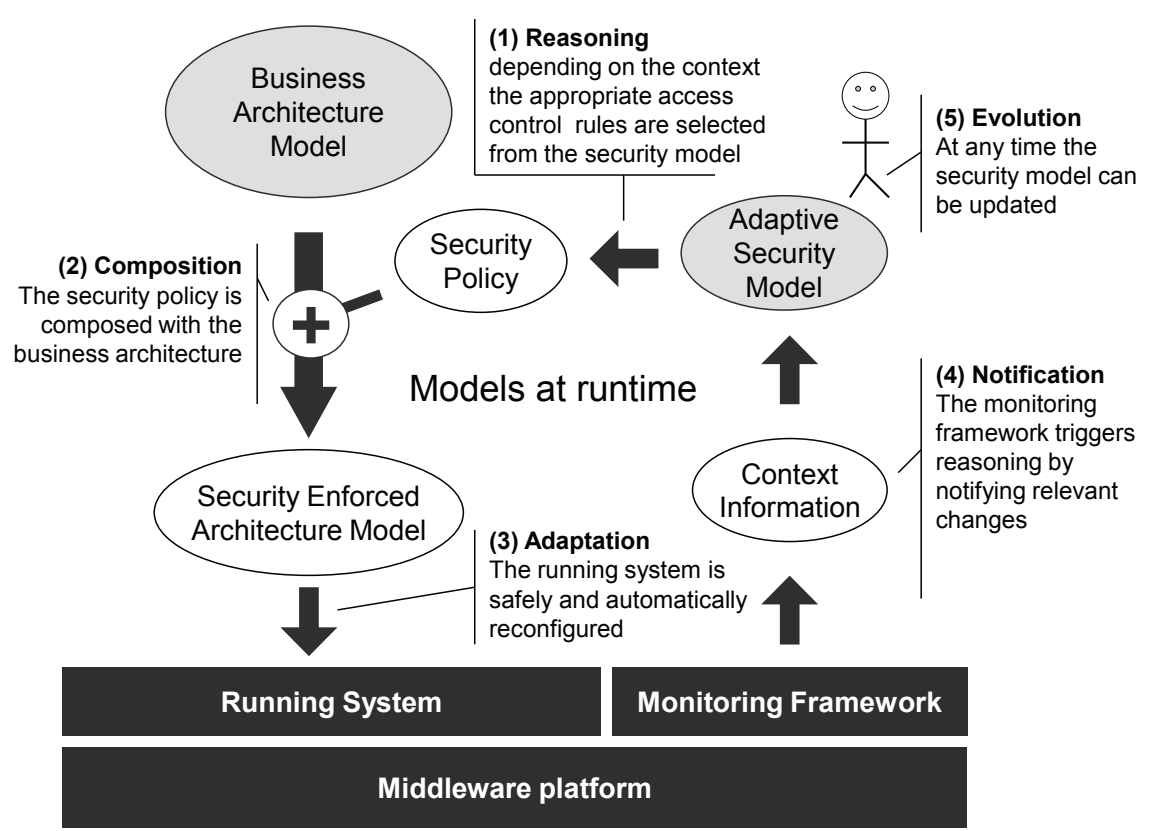

Fig. 1. Overview of the Model-Driven Security Approach of [24]

In [24], the authors have proposed to leverage MDE techniques to provide a very flexible approach for managing access control. The different steps of this approach are summed up in Figure 1. On one hand, access control policies are defined by security experts, using a DsmL, which describes the concepts of access control, as well as their relationships. On the other hand, the application is designed using another DsmL for describing the architecture of a system in terms of components and bindings. This component-based software architecture only contains the business components of the application, which encapsulate the functionalities of the system, without any security concern. Then, the authors define mappings between both Dsmls describing how security concepts are mapped to architectural concepts. These mappings are used to fully generate an architecture that enforces the security rules. When the security policy 
is updated, the architecture is also updated. Finally, the proposed technique leverages the notion of models@runtime [21] in order to keep the architectural model (itself synchronized with the access control model) synchronized with the running system. This way, the running system can be dynamically updated in order to reflect changes in the security policy. Only users who have the right to access a resource can actually access this resource.

\section{A Running Example}

In this section, we give a motivating example which will be used throughout the paper for describing the diverse characteristics of delegation and illustrating the various aspects of our approach.

Let us consider a Library Management System (LMS) providing library services with security concerns like access control and delegation management. There are two types of user account: personnel accounts (director, secretary, administrator and librarian) are managed by an administrator; and borrower accounts (lecturer and student) are managed by a secretary. The director of the library has the same accesses as a secretary, but additionally, he can also consult the personnel accounts. The librarian can consult the borrower accounts. A secretary can add new books in the LMS when they are delivered. Lecturers and students can borrow, reserve and return books, etc. In general, the library is organized with the following entities and security rules.

Roles (users): access rights (e.g. working days)

Director (Bill): consult personnel account, consult, create, update, and delete borrower account.

Secretary (Bob and Alice): consult, create, update, and delete borrower account, add book.

Administrator (Sam and Tom): consult, create, update, and delete personnel account.

Librarian (Jane and John): consult borrower account, find book by state, find book by keyword, report a book damaged, report a book repaired, fix a book.

Lecturer (Paul) and Student (Mary): find book by keyword, reserve, borrow and return book.

\section{Resources and actions to be protected}

Personnel Account: consult, create, update, and delete personnel account. Borrower Account: consult, create, update, and delete borrower account. Book: report a book damaged, report a book repaired, borrow a book, deliver a book, find book by keyword, find book by state, fix a book, reserve a book, return a book

In this organization, users may need to delegate some of their authorities to other users. For instance, the director may need the help of a secretary to 
replace him during his absence. A librarian may delegate his/her authorities to an administrator during a maintenance day.

It is possible to only specify role or action delegations by using the DSML described in [24]. For instance, a role delegation rule can be created to specify that Bill, the director (prior to his vacation) delegates his role to $B o b$, one of his secretaries. But it is impossible for Bill to define whether or not Bob can re-delegate the director role to someone else (in case $B o b$ is also absent for some reason). The role delegation of Bill to $B o b$ is also handled manually: it is enforced when Bill creates the delegation rule and only revoked when Bill deletes this rule. There is no way for Bill to define a temporary delegation where its active duration is automatically handled. Obviously the DsmL described in [24] is not expressive enough to specify complex characteristics of delegation.

There are many delegation situations that should be supported by the system. We give some delegation situations of the LMS as follows:

1. The director $(B i l l)$ delegates his role to a secretary $(B o b)$ during his vacation (the delegation is automatically activated at the start of his vacation and revoked at the end of his vacation).

2. A secretary (Alice) delegates her task/action of create borrower account to a librarian (Jane).

3. A secretary $(B o b)$ transfers his role to an administrator (Sam) during maintenance day. In case of a transfer delegation, the delegator temporarily loses his/her rights during the time of delegation.

4. The role administrator is not delegable.

5. The permission of deleting borrower account is not delegable.

6. The director can delegate, on behalf of a secretary, the secretary's role (or some his/her permitted actions) to a librarian (e.g. during the secretary's absence).

7. If a librarian empowered in role secretary by delegation is no longer able to perform this task, then he/she can delegate, again, this role to another librarian.

8. The secretary empowered in role director by delegation is not allowed to delegate/transfer, again, this role to another secretary.

9. A secretary is allowed to delegate his/her role to a librarian only and to one librarian at a given time.

10. A secretary is allowed to delegate his/her task of book delivery to a librarian only and scheduled on every Monday.

11. Bill can delegate his role and permitted actions only to $B o b$

12. $B o b$ is not allowed to delegate his role.

13. Alice is not allowed to delegate her permitted action of book delivery.

14. Users can always revoke their own delegations.

15. The director can revoke users from their delegated roles.

16. A secretary can revoke librarians empowered in secretary role by delegation, even if he/she is not the creator of this delegation (e.g. the creator is the director or another librarian).

This running example shows the two levels of delegation rules as defined in the previous section: user-level (rules defined by a user: e.g. situations $1,2,3$ ) and 
master-level (rules defined by a security officer: e.g. 4, 5, 6). Obviously, delegation rules at user-level have to conform to rules at master-level. For example, the security officer can define that users of role director are able to delegate on behalf of users of role secretary. Then at user-level, Bill (director) can create a delegation rule to delegate, on behalf of Alice, her role (secretary) to Jane (librarian).

\section{Model-Driven Adaptive Delegation}

\subsection{Overview of Our Approach}

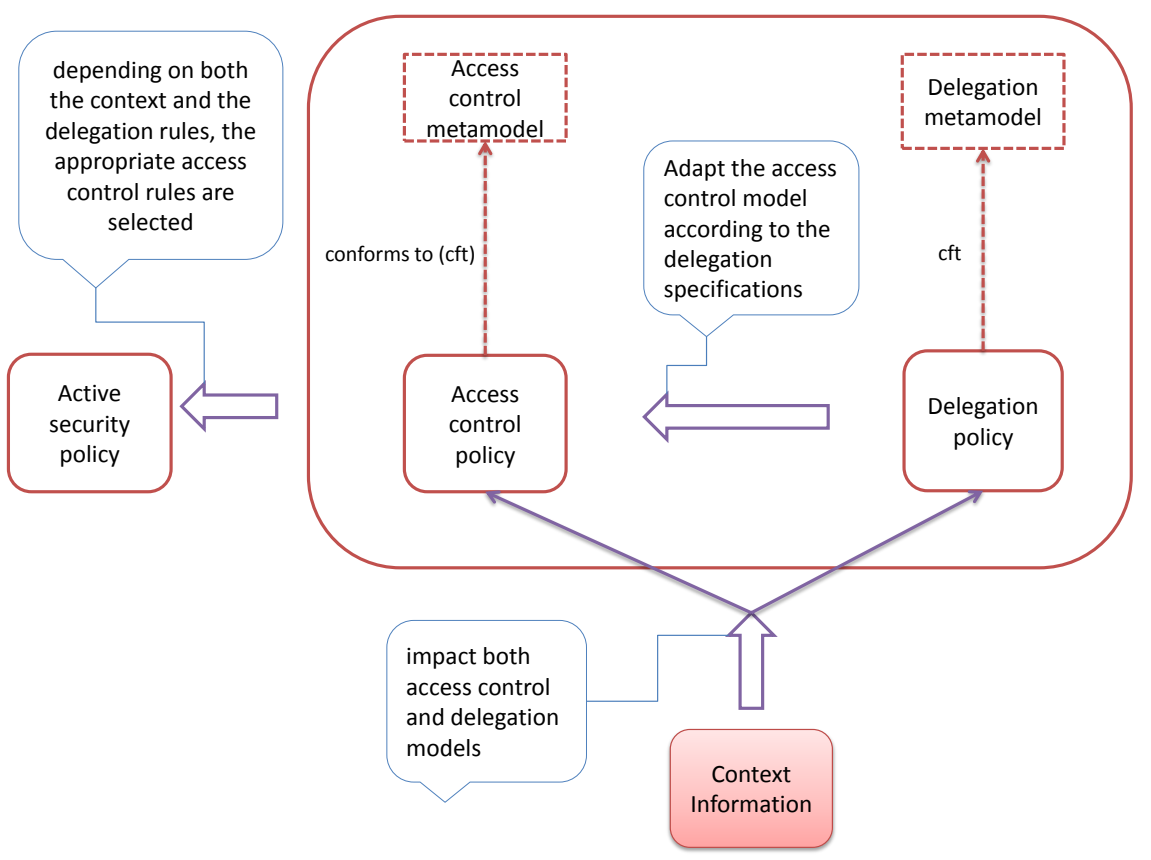

Fig. 2. Delegation impacting Access Control

In our approach, as noted in Section 2, delegation is considered as a "metalevel" mechanism which impacts the existing access control policies, like an aspect can impact a base program. We claim that to handle advanced delegation rules, an ideal solution is to logically separate the delegation rules from the access control policy, each being specified in isolation, and then compose/weave them together to obtain a new access control policy (called active security policy) reflecting the delegation-driven policy (Figure 2). We present our metamodel (DSML) for specifying delegation based on RBAC in Section 4.2. 


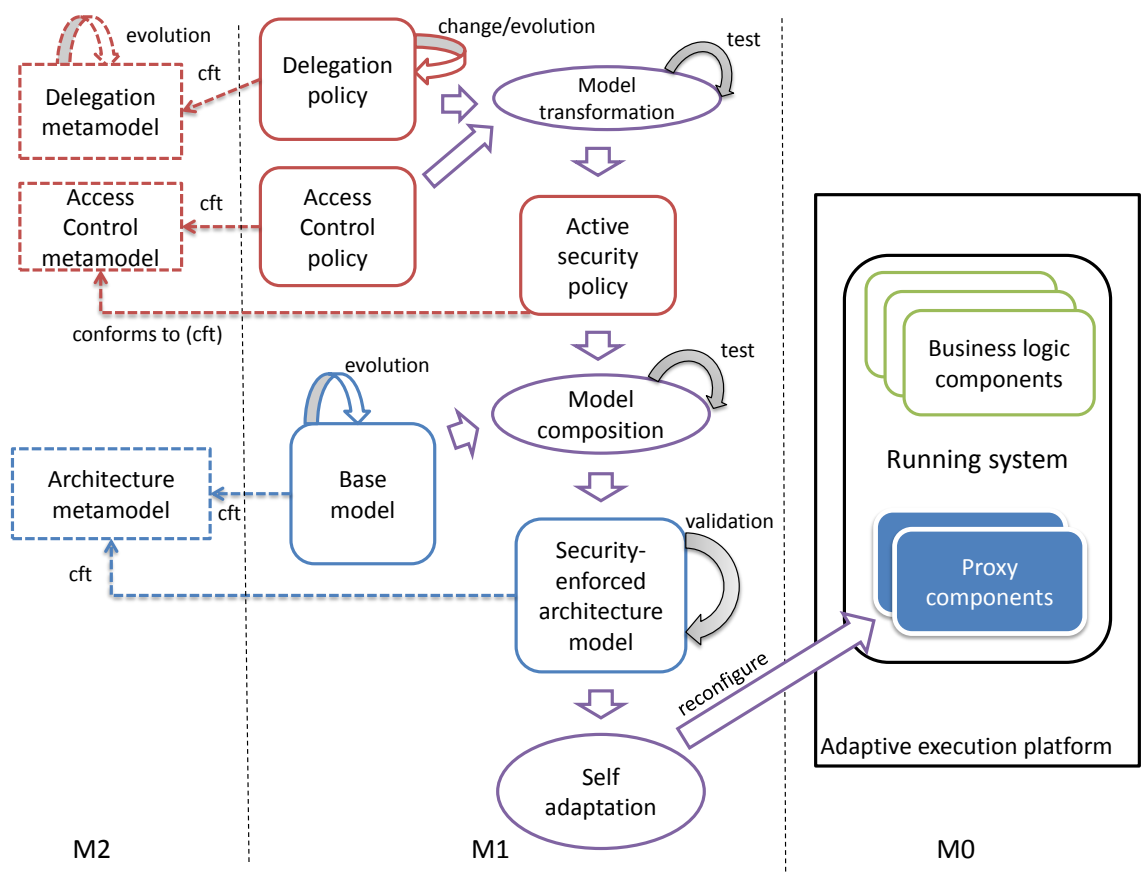

Fig. 3. Overview of our approach

The separation of concerns is not only between delegation and access control, but also between the security policy and the business logic of the system. Figure 3 presents a wider view of the overall approach. In order to enforce a security policy for the system, the core business architecture model of the system is composed with the active security policy previously obtained. The architecture model is expressed in another DsML, called architecture metamodel (an architecture modeling language described in [24]). The idea is to reflect security policy into the system at the architecture level. Section 4.3 defines transformation rules to show how security concepts are mapped into architectural concepts.

The security-enforced architecture model obtained above is a pure architecture model which by itself reflects how the security policy is enforced in the system. Our model-driven framework to reflect security policy at the architecture model is generic, meaning that from the security-enforced architecture model of the system, it is possible to enforce security policy for running systems on different execution platforms. In Section 5, we show how our approach is applied for two different adaptive execution platforms, i.e. OSGi [33] and Kevoree [13] 1. It is important to note that the security-enforced architecture model is not used for generating the whole system but only the proxy components. These proxy components can be adapted and integrated with the running system at runtime to physically enforce the security policy. The adaptation and integra-

\footnotetext{
${ }^{1}$ www.kevoree.org, last access October 2013
} 
tion can be done by leveraging the runtime adaptation mechanisms provided by modern adaptive execution middleware platforms. The approach of possibly generating proxy components overcomes some main limitations of [24]. Section 4.4 is dedicated to discuss our strategy for adaptation and evolution of the secure systems.

\subsection{Delegation Metamodel}

Our metamodel, displayed in Figure 4, defines the conceptual elements and their relationships that can be used to specify access control and delegation policies which are defined in Section 2. Because the delegation mechanism is based on RBAC, we first explain the main conceptual elements of role-based access control. Then, we show how our conceptual elements of delegation, based on the RBAC conceptual elements, can be used to specify various delegation features which are defined in Section 2.

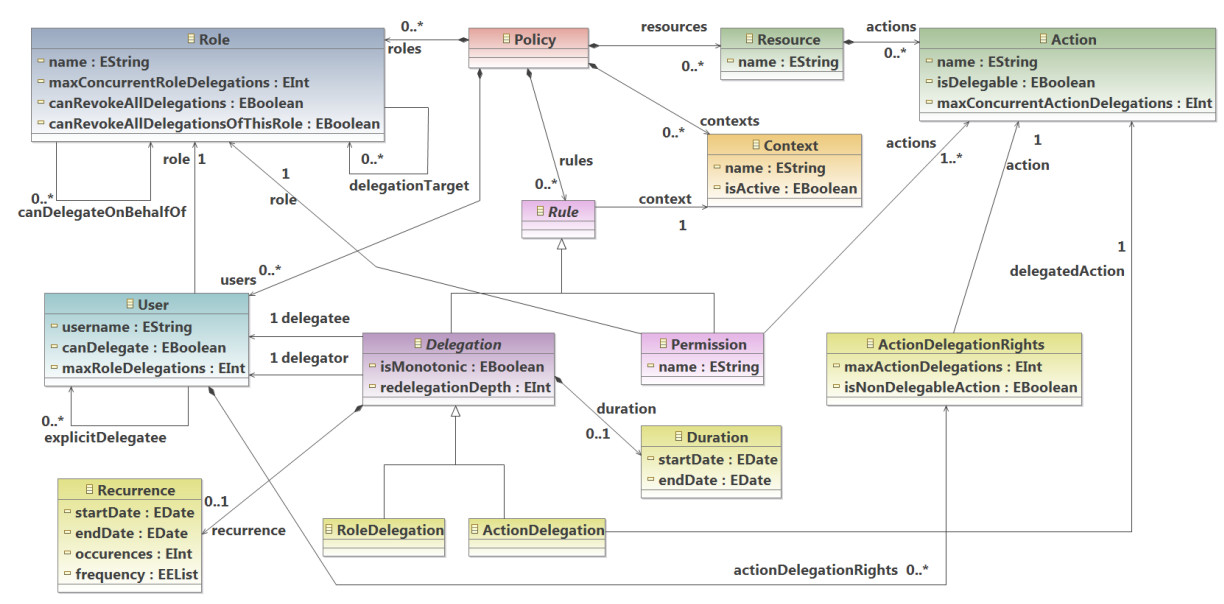

Fig. 4. The Delegation metamodel

As shown in Figure 4, the root element of our metamodel is the Policy. It contains Users, Roles, Resources, Rules, and Contexts. Each user has one role. A security officer can specify all the roles in the system, e.g. admin, director, etc., via the Role element. In order to specify an access control policy, the security officer should have defined in advance the resources that must be protected from unauthorized access. Each resource contains some actions which are only accessible to authorized users. These protections are defined in rules: permission rules and delegation rules. Permission rules are used to specify which actions are accessible to users based on their roles. That means, without delegation rules or user-specific access control rules, every user is able to access the actions associated with his/her role only. Delegation rules are used to specify which actions are accessible to users by delegation. There are two basic types of delegation: 
- Role delegation: When users empowered in role(s) delegated by other user(s), they are allowed to access not only actions associated with their roles but also actions associated with the delegated role(s).

- Action delegation: Instead of delegating their roles, users may want to delegate only some specific actions associated with their roles.

Another important aspect of our access control and delegation framework is the notion of context which has been introduced in Section 2.2. It can be seen from our metamodel that every permission/delegation rule is associated with a context. A rule is only active within its context. The concept of context actually provides our model with high flexibility. Security policies can be easily adapted according to different contexts.

The full metamodel for specifying delegation is displayed in Figure 4. It depicts the features that are supported by our delegation framework. All delegation management features are developed based on two basic types of delegation mentioned above. In the following, we show how the delegation features can be specified, w.r.t. our metamodel. In other words, this is how the formal definitions in Section 2 are actually implemented.

- Temporary delegation: This is one of the most common types of delegation used by users. It describes when the delegation starts to be active and when it ends. The delegator can specify that the delegated role/action is authorized only during a given time interval, e.g. situation 1 of the running example in Section 3. Actually, this can be specified using the recurrence of delegation described below, but we want to define it separately because of its common use.

- Monotonicity (Transfer of role or permissions): A property isMonotonic can be used to specify if a delegation is monotonic or non-monotonic. The former $($ isMonotonic $=$ true $)$ specifies that the delegated access right is available to both the delegator and delegatee after enforcing this delegation. As defined in Section 2, this delegation is called a grant delegation. The latter $($ isMonotonic $=$ false) means the delegated role/action is transferred to the delegatee, and the delegator temporarily loses his rights while delegating, e.g. situation 3. In this case, the delegation is called a transfer delegation.

- Recurrence: It refers to the repetition of the delegation. A user may want to delegate his role to someone else for instance every week on Monday. Recurrence defines how the delegation is repeated over time. It is similar to what is implemented in calendar system and more precisely the icalendar standard $\left(\mathrm{RFC} 2445^{2}\right)$. It has several properties; the startDate and endDate are the starting and ending dates of the recurrence. In addition, the startDate defines the first occurrence of the delegation. The frequency indicates one of the three predefined types of frequency, daily, weekly or monthly. The occurrences is the number of times to repeat the delegation. If the occurrences is for instance equals to 2 it means that it should only be repeated twice even

\footnotetext{
${ }^{2}$ http://www.rfc-editor.org/info/rfc2445
} 
when the endDate is not reached. An example of this delegation is situation 10 of the running example.

- Delegable roles/actions: These kinds of delegation define which roles or actions can be delegated and how (master-level). A policy officer can specify that a role can only be delegated/transferred to specific role(s), e.g. situation 9. If no delegation Target is defined for a role, this role cannot be delegated/transferred, e.g. situation 4. If a role or action (isDelegable $=$ false) is not delegable, it should never be included in a delegation rule. Moreover, a role can also be delegated by a user not having this role but his/her own role is specified as can delegate on behalf of a user in this role (canDelegateOnBehalfOf $=$ true), e.g. situation 6 .

- Multiple delegations: It should be possible to define the max number of concurrent delegations in which the same role or action can be delegated at a given time (master-level delegation rule). The properties maxConcurrentRoleDelegations and maxConcurrentActionDelegations define how many concurrent delegations of the same role/action can be granted, e.g. situation 9. Moreover, it is possible to define for each specific user a specific maximum number of concurrent delegations of the same role/action: maxRoleDelegations and maxActionDelegations.

- User specific delegation rights: All user-specific elements are used to define more strict rules for a specific user rather for his/her role. There are other user-specific delegations than maxRoleDelegations and maxActionDelegations. It is possible to define that a specific user is allowed to delegate his role/permitted action(s) or not (canDelegate $=$ true or false), e.g. situation 12. The property isNonDelegableAction specifies an action that a specific user cannot delegate, e.g. situation 13. Moreover, the security officer can define to which explicit user(s) only (explicitDelegatee) a user can delegate/transfer his role to, e.g. situation 11.

- Multi-step delegation: It provides flexibility in authority management, e.g. situations 7,8 . The property redelegationDepth is used to define whether or not the role/action of a delegation can be delegated again. When a creator creates a new delegation, he/she can specify how many times the delegated role/action can be re-delegated. If the redelegationDepth $=0$, it means that the role/action cannot be delegated anymore, e.g. situation 8 . If the redelegationDepth $>0$, that means the role/action can be delegated again and each time it is re-delegated, the redelegationDepth is decreased by 1 .

- Revocations: All users can revoke their own delegations, e.g. situation 14. Security officer may set canRevokeAllDelegations = true for a role with a super revocation power in such a way that a user empowered in this role can revoke all delegations, e.g. situation 15. Moreover, a role can also be defined such that every user empowered in this role can revoke any delegation from this role (canRevokeAllDelegationsOfThisRole $=$ true $)$, even he/she is not the delegator of the delegation, e.g. situation 16 .

Moreover, each possible instance of the security policy has to satisfy all necessary validation condition expressed as OCL invariants. For example, we can 
make sure that no delegation is out of target, meaning that delegatee's role has to be a delegation target of delegator's role:
context
Delegation
inv
NoDelegationOutOfTarget:

self.delegator.role.delegationTarget $->$ exists $(\mathrm{t} \mid \mathrm{t}=$ self.delegatee.role)

Or to check that for every user, the number of concurrent role delegations cannot be over its thresholds:

context User inv NoRoleDelegationOverMax: RoleDelega-

tion.allInstances $->$ select $(\mathrm{d} \mid$ d.delegator $=$ self $)->\operatorname{size}() \leq$ self.role.maxConcurrentRoleDelegations and RoleDelegation.allInstances $->$ select $(\mathrm{d} \mid$ d.delegator $=$ self $)->$ size ()$\leq$ self.maxRoleDelegations

Other examples are to restrict the value of the redelegationDepth must not be negative, or startDate cannot be later than endDate:

context Delegation inv NonNegativeDeleDepth: self.redelegationDepth $\geq 0$

context Duration inv ValidDates: self.startDate $\leq$ self.endDate

\subsection{Transformations/Compositions}

After specifying a security policy by the DsmL described in Section 4.2, it is crucial to dynamically enforce this policy into the running system. Transformations play an important role in the dynamic enforcement process. Via model transformations, security models containing delegation rules and access control rules are automatically transformed into component-based architecture models. Note that instances of security models and architecture models are checked before and after model transformations, using predefined OCL constraints.

The model transformation is executed according to a set of transformation rules. The purpose of defining transformation rules is to correctly reflect security policy at the architectural level. Based on transformation rules, security policy is automatically transformed to proxy components, which are then integrated to the business logic components of the system in order to enforce the security rules. The metamodel of component-based architecture can be found in [24] and an instance of it can be seen in Figure 7. We first describe the transformation that derives an access control model according to delegation rules (step 1), and then describe another transformation to show how security policy can be reflected at the architecture level (step 2). Moreover, we also show an alternative way of transformation that combines two steps into one.

Adapting Role-Based Access Control policy model to reflect delegation (step 1): Within the security model shown in Figure 2, delegation rules are considered as "meta-level" mechanisms that impact the access control rules. The appropriate access control rules and delegation rules are selected depending on the context information and/or the request of changing security rules coming from the system at runtime. According to the currently active context (e.g. WorkingDays), only in-context delegation rules and in-context access control rules of the security model (e.g. rules that are defined with context $=$ WorkingDays) are taken into account to derive the active security policy model (Figure 


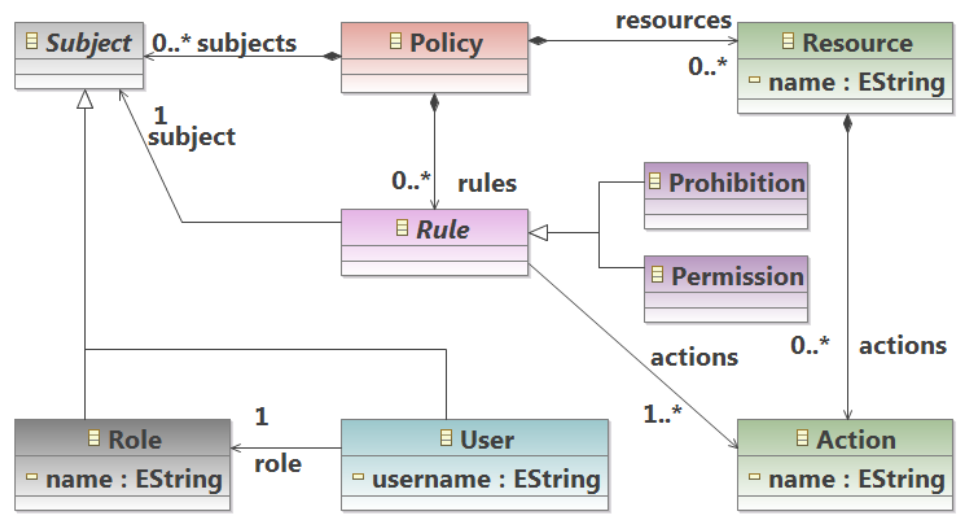

Fig. 5. A pure RBAC metamodel

2 ). Theoretically, we could say that delegation rules impact the core RBAC elements in the security model in order to derive a pure RBAC model (without any delegation and context elements) which conforms to a "pure" metamodel of RBAC (Figure 5). Delegation elements of a security policy model are transformed as follows:

A.1: Each action delegation is transformed into a new permission rule. The subject of the permission is user (delegatee) object. The set of actions of the permission contains the delegated action.

A.2: Each role delegation is transformed as follows. First, a set of actions associated to a role is identified from the permissions of this role. Then, each action is transformed into a permission like transforming an action delegation described above.

A.3: A temporary delegation is only taken into account in the transformation if it is in active duration defined by the start and end properties. In fact, when its active duration starts the (temporary) action/role delegation is transformed into permission rule(s) as described above. When its active duration ends the temporary delegation is removed from the policy model.

A.4: If an action delegation is of type transfer delegation (monotonic), then it is transformed into a permission rule and a prohibition rule. The subject of the permission is the user-delegatee object. The set of actions of the permission contains the delegated action. The subject of the prohibition is the user-delegator object. The set of actions of the prohibition contains the delegated action.

A.5: If a role delegation is of type transfer delegation, then it is also transformed into a permission rule and a prohibition rule. The subject of the permission is the user-delegatee object. The set of actions of the permission contains the delegated actions. The delegated actions here are the actions associated with this role. The subject of the prohibition is the user-delegator object. The set of actions of the prohibition also contains the delegated actions.

A.6: If a delegation rule is defined with a recurrence, based on the values set 
to the recurrence, the delegation rule is only taken into account in the transformation within its fromDate and untilDate, repeated by frequency and limited by occurrences. In other words, only active (during recurrence) delegation rules are transformed.

A.7: (User-specific) If a user is associated with any non-delegable action, the action delegation containing this action and this user (as delegator) is not transformed into a permission rule. Similarly, if a user is specified as he/she cannot delegate his/her role/action, no role/action delegation involving this user is transformed.

A.8: (Role/action-specific) Any delegation rule with a non-delegable role/action will not be transformed. In fact, a delegation rule is only transformed if it satisfies (at least) both user-specific and role/action-specific requirements.

A.9: Only a role delegation to a user (delegatee) whose role is in the set of delegationTarget will be considered in the transformation.

A.10: Before any delegation is taken into account in the transformation, it has to satisfy the requirements of max concurrent action/role delegations. Note that the user-specific values have higher priorities than the role-specific values.

A.11: A delegation is only transformed if its redelegationDepth $>0$. Whenever a user empowered in a role/an action by delegation re-delegates this role/action, the newly created delegation is assigned a redelegationDepth $=$ the previous redelegationDepth - 1 .

After transforming all delegation rules, we obtain a pure RBAC model which reflects both the delegation model and access control model. This pure RBAC model is then transformed into a security-enforced architecture model as described next.

Transformation of Security Policy to Component-based Architecture (step 2): The transformation rules are defined below. The goal is to transform every security policy model (pure RBAC model obtained in step 1) which conforms to the metamodel shown in Figure 5 to a component-based architecture model which conforms to the metamodel described in [24]. However, both the security policy model and the base model provided by a system designer are used as inputs for the model transformation/composition. Via a graphical editor, the security designer must define in advance how the resource elements in the policy model are related to the business components in the base model. Figure 6 shows how each action in the policy can be mapped to the Java method in the business logic.

Because the base model already conforms to the architecture metamodel, we now only focus on transforming the security policy model into the securityreflected architecture model. As we know, this transformation/composition process will also weave the security-reflected elements into the base model in order to obtain the security-enforced architecture model.

The core elements of RBAC like resource, role, and user are transformed following these transformation rules. All the transformation rules make sure that the security policy is reflected at the architectural level. 


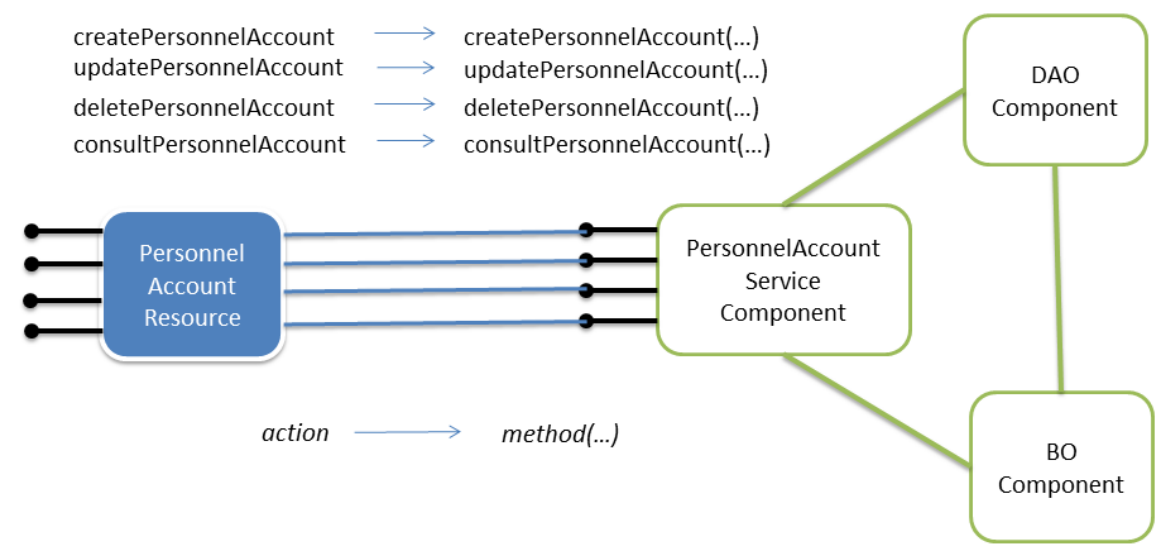

Fig. 6. Mapping Resources to Business Logic Components

R-A.1: Each resource is transformed into a component instance, called a resource proxy component. According to the relationship between the resource elements in the policy model and the business components in the base model, each resource proxy component is connected to a set of business components via bindings. To be more specific, each action of a resource element is linked to an operation of a business component (Figure 6). By connecting to business components, a resource proxy component provides and requires all the services (actions) offered by the resource.

R-A.2: Each role is also transformed into a role proxy component. According to the granted accesses (permission rules associated with this role) to the services provided by the resources, the corresponding role proxy component is connected to some resource proxy component(s) (Figure 7). A role proxy component is connected to a resource proxy component by transforming granted accesses into ports and bindings. Each (active) access granted to a role is transformed into a pair of ports: a client port associated with the role proxy component, a server port associated with the resource proxy component, and a binding linking these ports.

R-A.3: Each user element defined in the policy model is also transformed into a user proxy component. Because each user must have one role, each user proxy component is connected to the corresponding role proxy component. However, each user may have access to actions associated to not only his/her role but also to actions associated to other roles by delegation. Thus, each user proxy component may connect to several role proxy components. The connection is established by transforming each access granted to a user into a pair of ports: a client port associated with the user proxy component, a server port associated with the corresponding role proxy component (providing the access/port), and a binding linking these ports (Figure 7). Actually, the granted accesses are cal- 


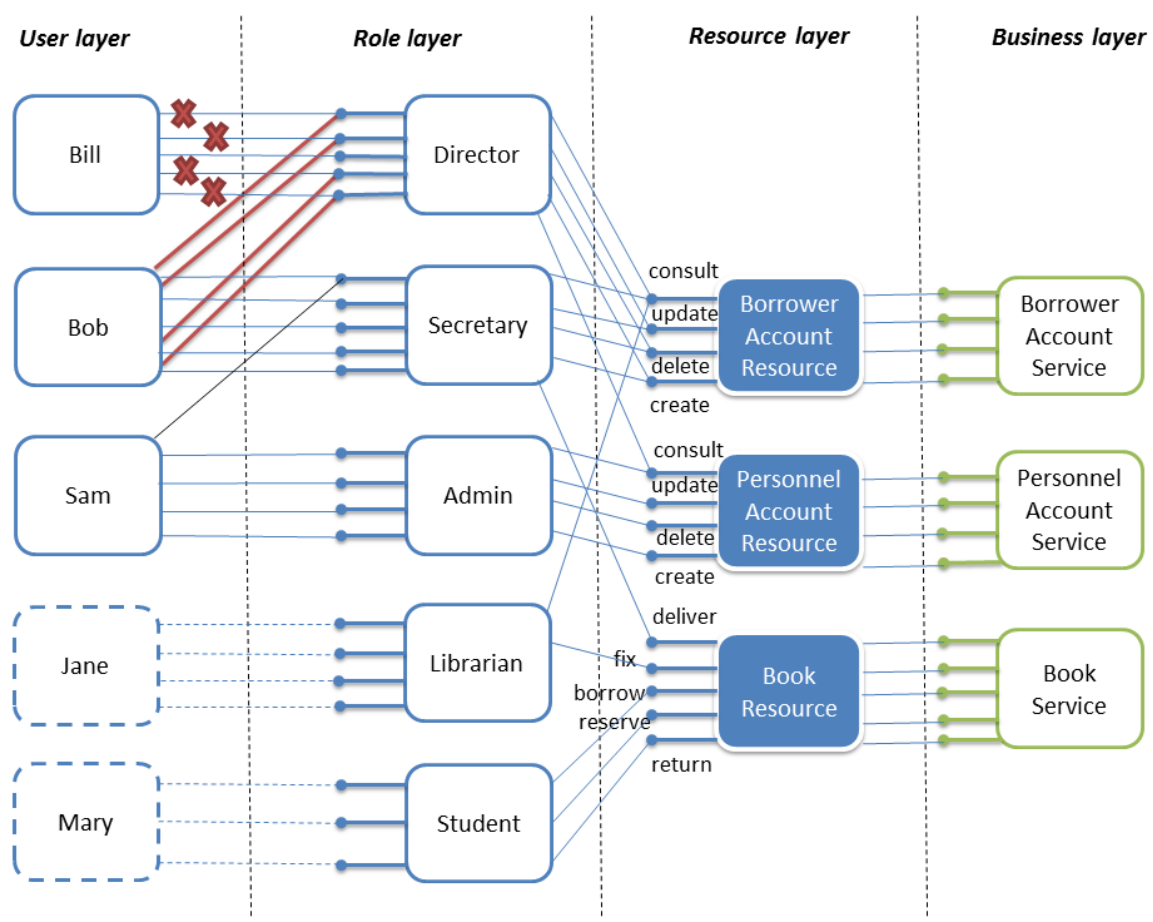

Fig. 7. Architecture reflecting security policy before and after adding a delegation rule (bold lines)

culated not only from permission rules but also from prohibition rules. Simply, the granted accesses that equal permissions exclude prohibitions.

In our approach, revocation of a delegation simply consists in deleting the corresponding delegation rule. In this way, the revocation is reflected at the architectural level and physically enforced in the running system. Moreover, both the delegator and delegatee elements will be removed if these users are not involved in any delegation rules. As described above, user elements are transformed into proxy components. However, it is important to stress that only users involved in delegation rules (e.g. Bill, Bob and Sam in Figure 7) are created in the security policy model and transformed into proxy components. Users who are not involved in any delegation rules (e.g. Jane and Mary in Figure 7), are manipulated as session objects which directly access the services offered by the corresponding role proxy components.

Two steps described above are two separate model transformations that are mainly used to explain how delegation can be considered as a "meta-level" mechanism for administrating access rights. The first model transformation is to transform a delegation-driven security model into a pure RBAC model. The second model transformation is to transform the RBAC model into an architecture model. In fact, these two steps could be done in only one model transforma- 
tion that directly transforms the delegations, the access control policy and the business logic model into an architecture model reflecting the security policy. However, this alternative way (described in the following) has the disadvantage of losing the intermediate security model (the active security policy) that could be useful for traceability purpose.

An alternative way using only one transformation: In this approach, we have to define different transformation rules to transform directly every security policy model which conforms to the metamodel, shown in Figure 4, to a component-based architecture model which conforms to the architecture metamodel described in [24]. Core elements of RBAC like resources, roles, and users are transformed following these transformation rules:

R-B.1: Each resource is transformed into a component instance, called a resource proxy component (already presented).

R-B.2: Each role also is transformed into a role proxy component (already presented). The only difference here is that the context has to be taken into account (in the step 2 of transformation mentioned earlier, no context existed because context was already dealt with in the step 1). Because every permission is associated with a context, we only transform permissions with the context that is active at the moment.

R-B.3: Each user element defined in the policy model is also transformed into a user proxy component. However, the connection (via bindings) from a user proxy component to the role proxy component(s) is not only depended on the user's role but also delegation rules that the corresponding user involved in. The transformation of delegation rules is presented below.

All the transformation rules above make sure that access control rules are reflected at the architecture level. However, the delegation rules will impact this transformation process in order to derive the security-enforced architecture model reflecting both access control and delegation policy. Delegation elements of a policy model are transformed as follows:

R-B.4: Each action involved in an action delegation is transformed into a pair of ports and a binding. A client port (representing the required action) is associated with the user (delegatee) proxy component. The binding links the client port to the corresponding server port (representing the same action provided) that associated with the role proxy component reflecting the role of the delegator.

R-B.5: Each role delegation is transformed in a similar way as action delegation. First, a set of actions associated to a role can be identified from the permissions of this role. Then, each action in the set is transformed into a pair of ports and a binding as transforming an action delegation.

R-B.6: A temporary delegation is only transformed into bindings if it is still in active duration defined by start and end properties.

R-B.7: If a delegation is of type transfer delegation, then both user elements (delegator and delegatee) are transformed into delegator and delegatee proxy components as described above. The delegator proxy component is not 
connected to the corresponding role proxy component because he/she already transfered his/her access rights to the delegatee. Figure 7 shows a change in the architecture when Bill transfers his role to Bob.

R-B.8: If a delegation is defined with a recurrence, based on the values set to recurrence, the delegation rule is only active during the recurrence (similar to A.6).

R-B.9: If a user is associated with any non-delegable action, the delegation of this action is not taken into account while doing the transformation. Similarly, if a user is specified as he/she can not delegate his/her role/action, no delegation requested by this user will be transformed.

R-B.10: Only a role delegation to a user (delegatee) whose role is in the set of delegationTarget will be consider in the transformation.

R-B.11: Before any delegation is taken into account in the transformation, it has to satisfy the requirements of max concurrent action/role delegations. Note that the user-specific values have higher priorities than the role-specific values.

R-B.12: A delegation is only transformed if its redelegationDepth $>0$. Whenever a user empowered in a role/an action by delegation re-delegates this role/action, the newly created delegation is assigned a redelegationDepth $=$ the previous redelegationDepth -1 .

By taking into account delegation rules while transforming access control rules of policy model into security-enforced architecture model, both delegation and access control rules are reflected at the architecture level.

\subsection{Adaptation and Evolution Strategies}

The model transformation/composition presented in Section 4.3 ensures that the security policies are correctly and automatically reflected in an architectural model of the system. The key steps to support delegation (i.e. specifications and transformations) are already presented in Sections 4.2 and 4.3. The last step consists in a physical enforcement of the security policy by means of a dynamic adaptation of the running system. In this section, our adaptation and evolution strategies are discussed.

Adaptation The input for the adaptation process is a newly created securityenforced architecture model (Figure 8). First, this new architecture model is validated using invariant checking [22]. This valid architectural model actually represents the new system state the runtime must reach to enforce the new security policy of the system. According to the classical MAPE control loop of self-adaptive applications, our reasoning process performs a comparison (using EMFCommpare) between the new architecture model (target configuration) and the current architecture model (kept synchronized with the running system) [23]. This process triggers a code generation/compilation process, and also generates a safe sequence of reconfiguration commands [22]. Actually, the code generation/compilation process is only triggered if there are new proxy components, e.g. 


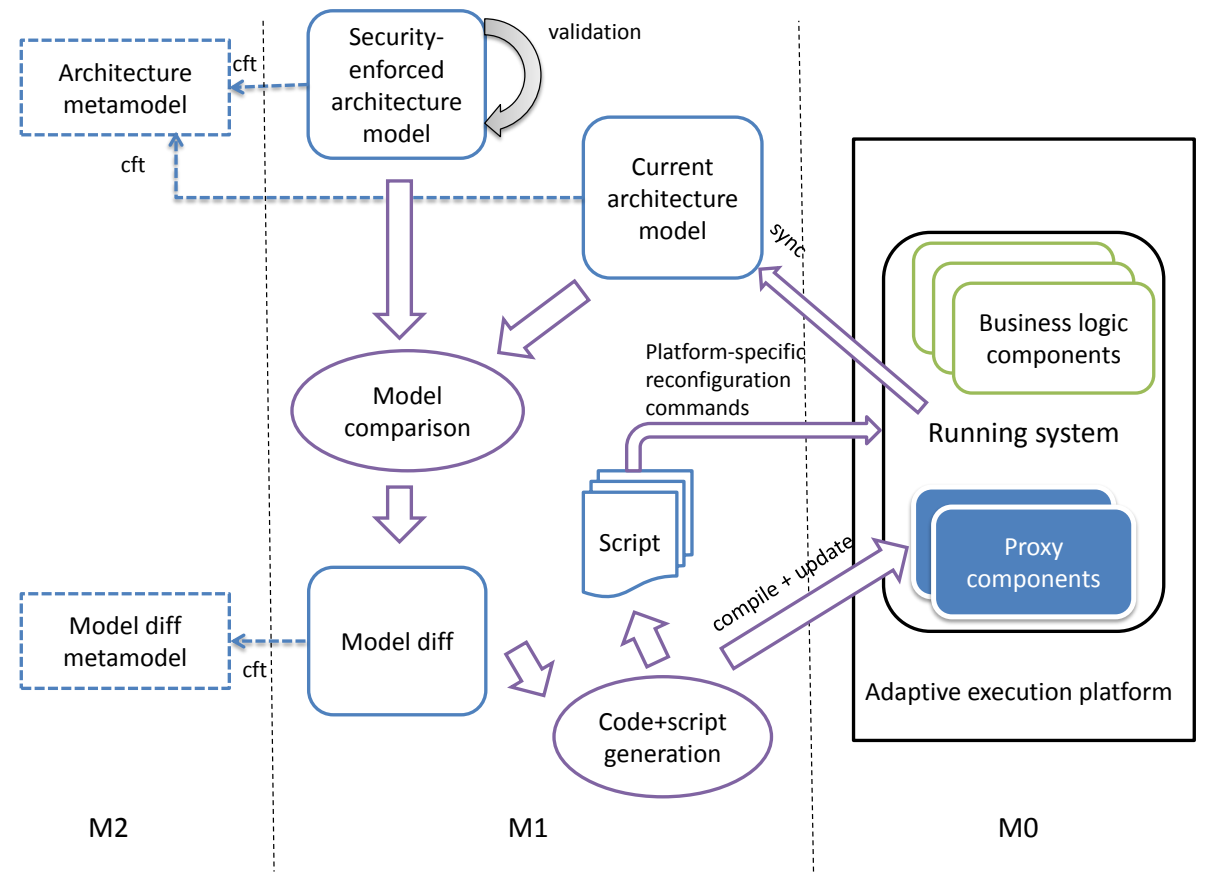

Fig. 8. Overview of our adaptation strategy

new user proxy components involved in delegation, that need to be introduced into the running system. The dynamic adaptation of the running system is possible thanks to modern adaptive execution platforms like OSGi [33] or Fractal [8], and most recently Kevoree [13], which provide low-level APIs to reconfigure a system at runtime. The running system is then reconfigured by executing the safe sequence of commands, compliant to the platform API, issued by the reasoning process. In an optimized model@runtime platform like Kevoree, all we need to do is to provide the reconfiguration script (Kevoree script) for the platform. The reasoning process is taken care of by the platform. In fact, the generation/compilation phase if needed could be time consuming. However, this phase has no impact on the running system, which remains stable until being adapted by executing the reconfiguration script. Thus, the actual adaptation phase lasts for only several milliseconds.

In [24], the adaptation is entirely based on executing platform-specific reconfiguration scripts specifying which components have to be stopped, which components and/or bindings should be added and/or removed. This results in several limitations regarding delegation mechanisms:

L.1: Using only reconfiguration scripts implies to create all the potentially needed ports (used for bindings between user proxy components) beforehand. But all the combinations of users, roles, resources, actions could lead to a com- 
binatorial explosion and make it infeasible for implementation.

L.2: In [24], the delegation between users are reflected using bindings connecting one user proxy component to another. But this approach is not suitable for supporting complex delegation features. For example, a transfer delegation will be reflected by adding bindings between the delegator and delegatee but removing bindings between delegator and the corresponding role proxy component. Consequently both delegator and delegatee cannot access the resource, which does not correctly reflect a transfer delegation.

L.1 can be solved by the automatic re-generation of proxy components and bindings between them according to changes in the architectural model. Moreover, as mentioned in Section 4.3, only users involved in a delegation are transformed into user proxy components with necessary ports and bindings. In this way, only required ports and bindings are created dynamically. L.2 is solved by our model transformation approach. All complex delegation features are considered as "meta-level" mechanisms that impact access control rules. In this way, a transfer delegation will be reflected by adding bindings between the delegatee and the corresponding delegated role proxy component, but removing bindings between delegator and the corresponding role proxy component.

Our adaptation strategy could take more time than simply running a reconfiguration script because of the generation and compilation time of newly generated proxy components. But the process of generating and compiling new proxy components does not in fact harm the performance because each proxy component is very light-weight and only necessary proxy components are generated (see Section 5). Moreover, for each specific security policy, it is possible to think in advance and prepare as many proxy component types as possible. This strategy could make the generation/compilation phase unnecessary for most of the cases, except some major evolution of the business logic and/or the security policy.

Evolution In [24], the evolution of the security policy is not totally dealt with. It is possible to run a reconfiguration script to reflect changes like adding, removing and updating rules. But adding a new user, role or resource requires the generation and compilation of new proxy components, which is impossible using only reconfiguration scripts. Thus, our strategy of automatically generating and compiling proxy components (see Section 5) is more practical w.r.t. evolution.

Another important aspect of evolution relates to the addition, removal or update of resources and actions in the business logic. The base architecture model can be updated with changes in the business logic, e.g. when a new resource is added. On the other side, security officers can manually update the mappings (Figure 6) following changes of resources/actions in the base architecture model. By composing the security model with the base architecture model as described earlier, the security policy is evolved together with the business logic of the system. 


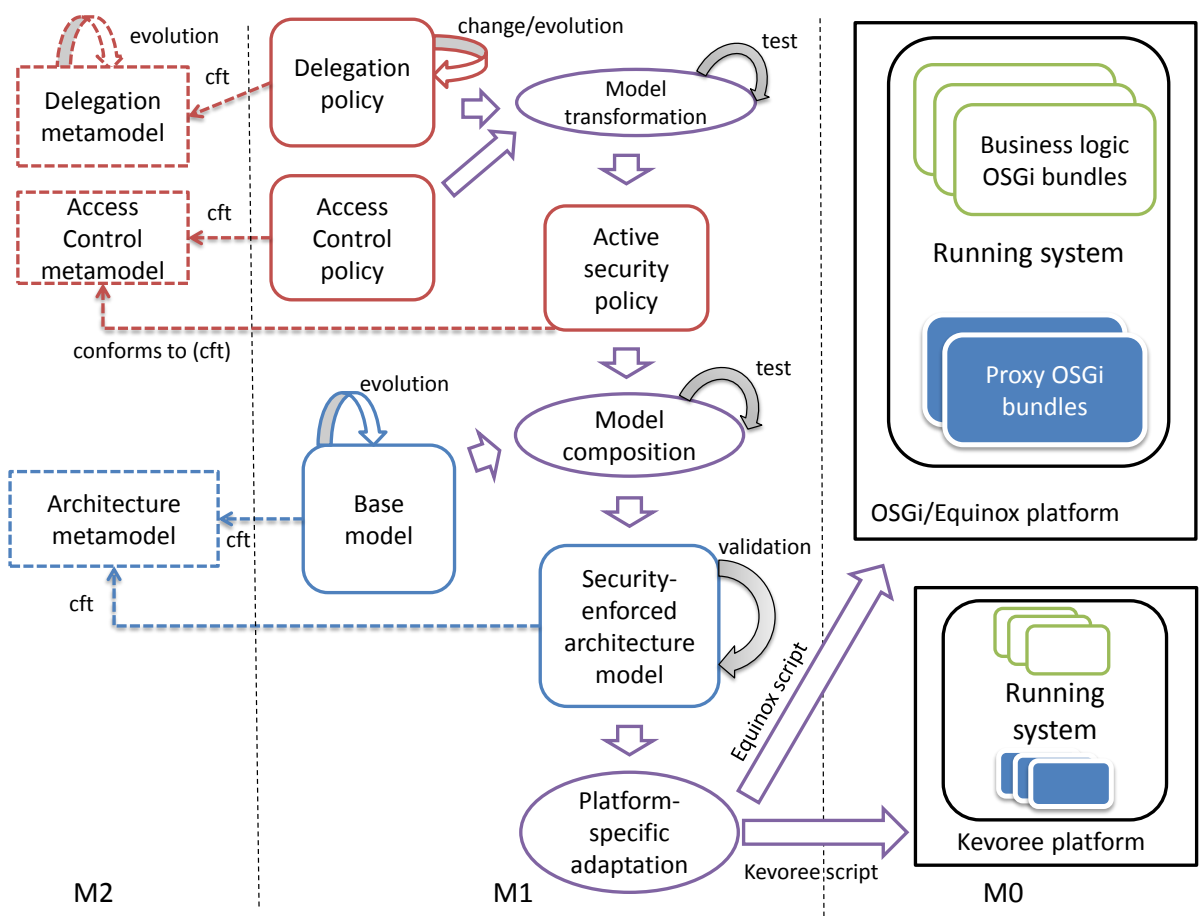

Fig. 9. OSGi and Kevoree as adaptive execution platforms

\section{Implementation and Evaluation}

This section shows how the steps described in Figure 3 have been implemented. In order to prove that our approach is generic, we target two different adaptive execution platforms: OSGi (Section 5.1) and Kevoree (Section 5.2). Figure 9 shows that our metamodels and model-to-model transformation/composition are generic, i.e. independent of execution platforms. Only the adaptation process (e.g. the reconfiguration script) and the running system are platform-specific. We evaluate our proof-of-concept implementations and discuss the results in Section 5.3. The description of three case studies used in our experiments are given below. The business logic of these case studies are the same for the OSGi and Kevoree adaptive execution platforms.

To evaluate the feasibility of our approach, we have applied it on three different Java-based case studies, which have also been used in our previous research work on access control testing [25]:

1) LMS: as described in our running example.

2) VMS $^{3}$ : The Virtual Meeting System offers simplified web conference services. The virtual meeting server allows the organization of work meetings on

\footnotetext{
${ }^{3}$ For more information about VMS (server side), please refer to http://franck.fleurey.free.fr/VirtualMeeting.
} 
a distributed platform. When connected to the server, a user can enter (exit) a meeting, ask to speak, eventually speak, or plan new meetings. There are three resources (Meeting, Personnel Account, User Account) and six roles (Administrator, Webmaster, Owner, Moderator, Attendee, and Non-attendee) defined for this system with many access control rules, and delegation situations between the users of each role.

3) ASMS: The Auction Sale Management System allows users to buy and sell products online. Each user in the system has a profile including some personal information. Users wanting to sell a product (sellers) are able to start a new auction by submitting a description of the product, the starting and ending date of the auction. There are five resources (Sale, Bid, Comment, Personnel Account, User Account) and five roles (Administrator, Moderator, Seller, Senior Buyer, and Junior Buyer) defined for this system, also with many access control rules, and delegation situations between users of each role.

Table 1. Size of each system in terms of source code

\begin{tabular}{|l|r|r|r|}
\hline & \# Classes & \# Methods & \# LOC \\
\hline LMS & 62 & 335 & 3204 \\
\hline VMS & 134 & 581 & 6077 \\
\hline ASMS & 122 & 797 & 10703 \\
\hline
\end{tabular}

Table 2. Security rules defined for each system

\begin{tabular}{|l|r|r|r|}
\hline & \# AC rules & \# Delegations & Total \\
\hline LMS & 23 & 4 & 27 \\
\hline VMS & 36 & 8 & 44 \\
\hline ASMS & 89 & 8 & 97 \\
\hline
\end{tabular}

Table 1 provides some information about the size of these three systems (the number of classes, methods and lines of code). In terms of security policies, Table 2 shows the number of access control (AC) rules and delegation rules defined for each system, used in our experiments.

All these systems are designed as component-based systems. The business components of each system contain the business logic, e.g. Book Service component, Personnel Account component, Meeting, Sale, Authenticate component, Data Access Object components, etc. To enable dynamic security enforcement for a system, the resources (components that have to be controlled) are specified in the base model, and mapped to the resources of the security policies. Our metamodels are applicable for different systems without any modification or adaptation. The structure of delegation and access control policies for all 
case studies is the same, only roles, users, resources, actions are specific to each case study. The proxy components are automatically generated and synchronized with the security policy model via model transformations and reconfiguration at runtime. The model-to-model transformation and model-to-text transformation (code generation) can be implemented correspondingly using transformation engines like Kermeta [26] (or $\mathrm{ATL}^{4}$ ), and Xpand [18].

\subsection{OSGi (Equinox) as the target Adaptive Execution Platform}

As shown in Figure 9, once we obtain the security-enforced architecture model from the previous steps, we have to reflect this security enforcement in the running system. In case we use Equinox ${ }^{5}$ as the target execution platform, all components (business logic components and proxy components) are implemented as OSGi bundles (Spring Dynamic Modules) [30]. In OSGi service platforms, there are two ways to declare and bind services via interfaces (ports): declaring/binding exported services in Spring osgi-context.xml files, or in the source code by overriding the method start of BundleActivator class of OSGi bundle. Here we show the code for the sake of simplicity but in practice, the declaration of services and bindings can be configured in XML files which means no need to recompile code to change the bindings. Once the services are made available, they can be called from other services. For example, the code snippet in Listing 1.1 shows how the deleteBorrowerAccountService of a proxy component of Role Director is bound to the exported service reference of the deleteBorrowerAccountService of the BorrowerAccountResource proxy component (lines 1-8). The lines 12-20 show that this Role Director can also access to consultPersonnelAccount of PersonnelAccountResource.

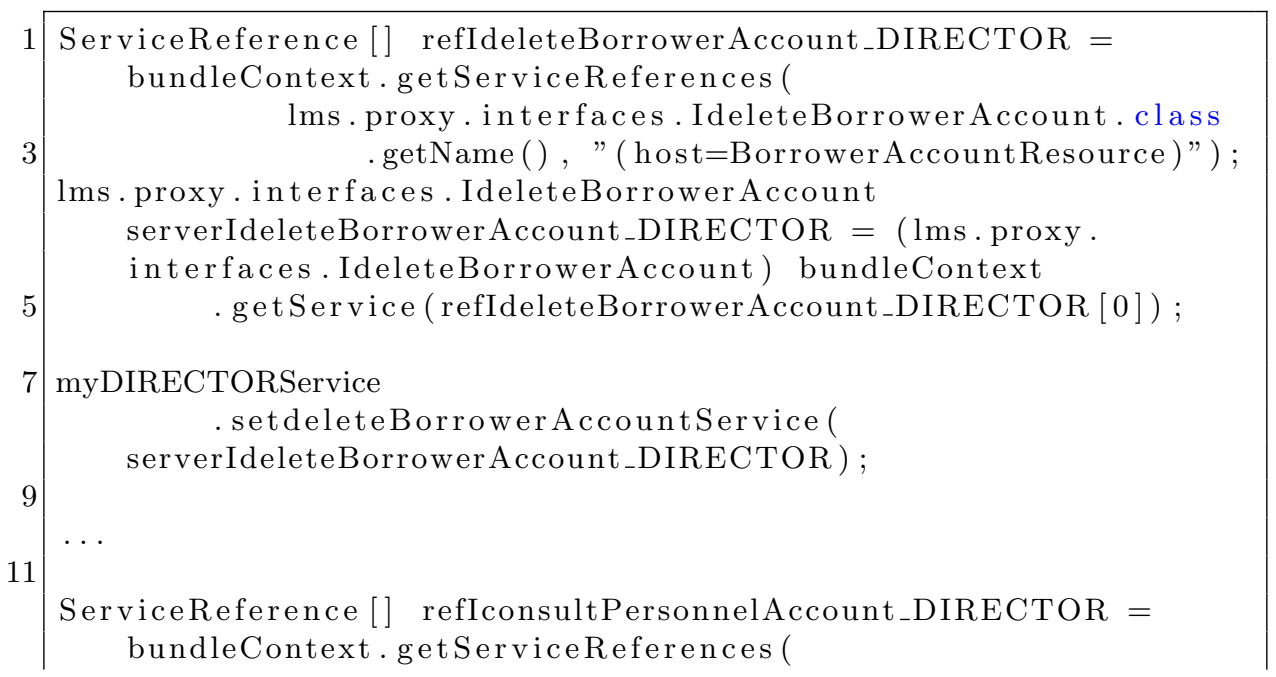

\footnotetext{
${ }^{4}$ http://www.eclipse.org/atl/

${ }^{5}$ http://www.eclipse.org/equinox/
} 


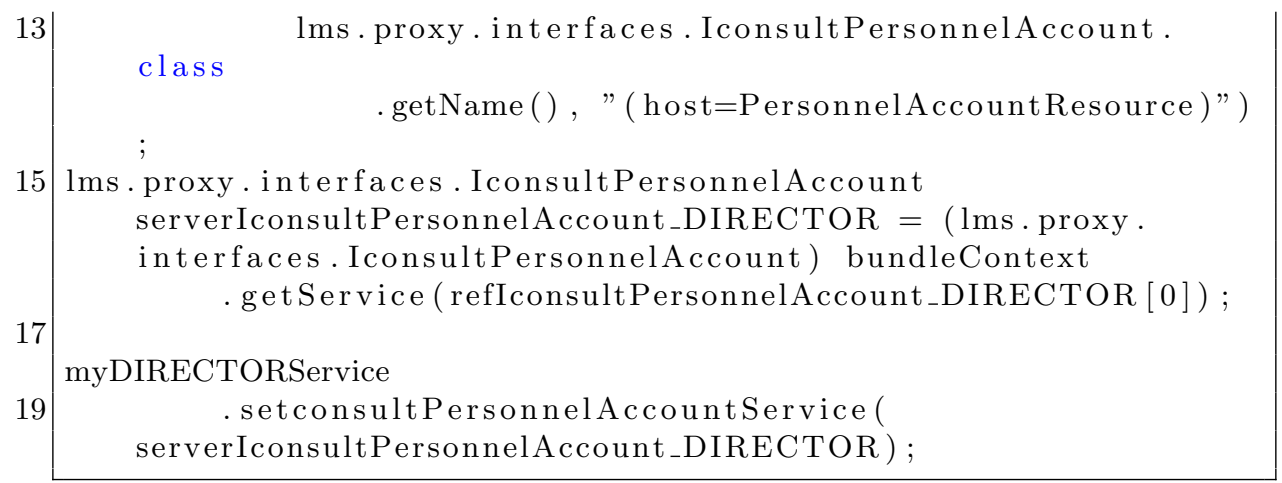

Listing 1.1. Services and Bindings in the Director proxy component

As we mentioned before, all the proxy components are very light-weight components. Every method of proxy components only contains the redirecting call to another service that (directly/indirectly) calls to the real method in the business logic. The code snippet in Listing 1.2 shows that a call to the deleteBorrowerAccount method (line 1) of a proxy component of Role Director actually is redirected to call the deleteBorrowerAccount method (line 3) of the BorrowerAccountResource proxy component that already was made available previously (lines 1-8, Listing 1.1). Similarly, the consultPersonnelAccount method (line 7) contains a call to the consultPersonnelAccount method (line 10) of the PersonnelAccountResource proxy component that already was made available previously (lines 12-20, Listing 1.1).

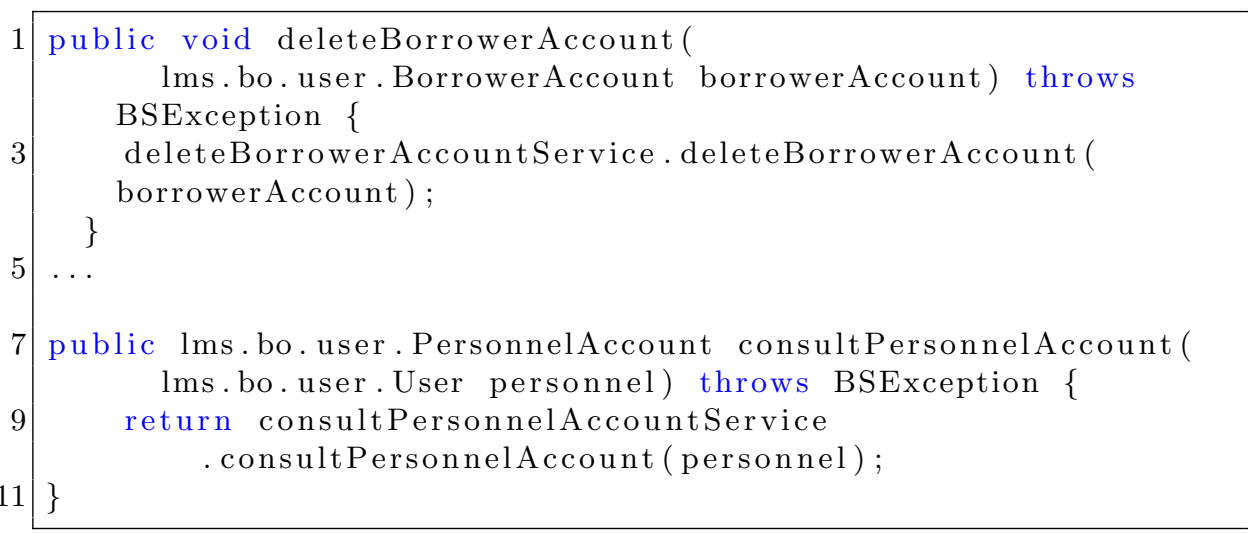

Listing 1.2. Redirecting the method calls in the Director proxy component

The adaptation process is directed by a generated reconfiguration script that is specific for Equinox adaptive execution platform. The reconfiguration script is executed in order to reflect the change of the policy from the model level to the running system, e.g. a new delegation rule is active. Figure 10 shows a new delegation rule has been enforced in the running system so that Bob (Secretary) is delegated the permission to consult personnel account by Bill (Director). This means after enforcing this delegation rule, there exists a connection from the port 


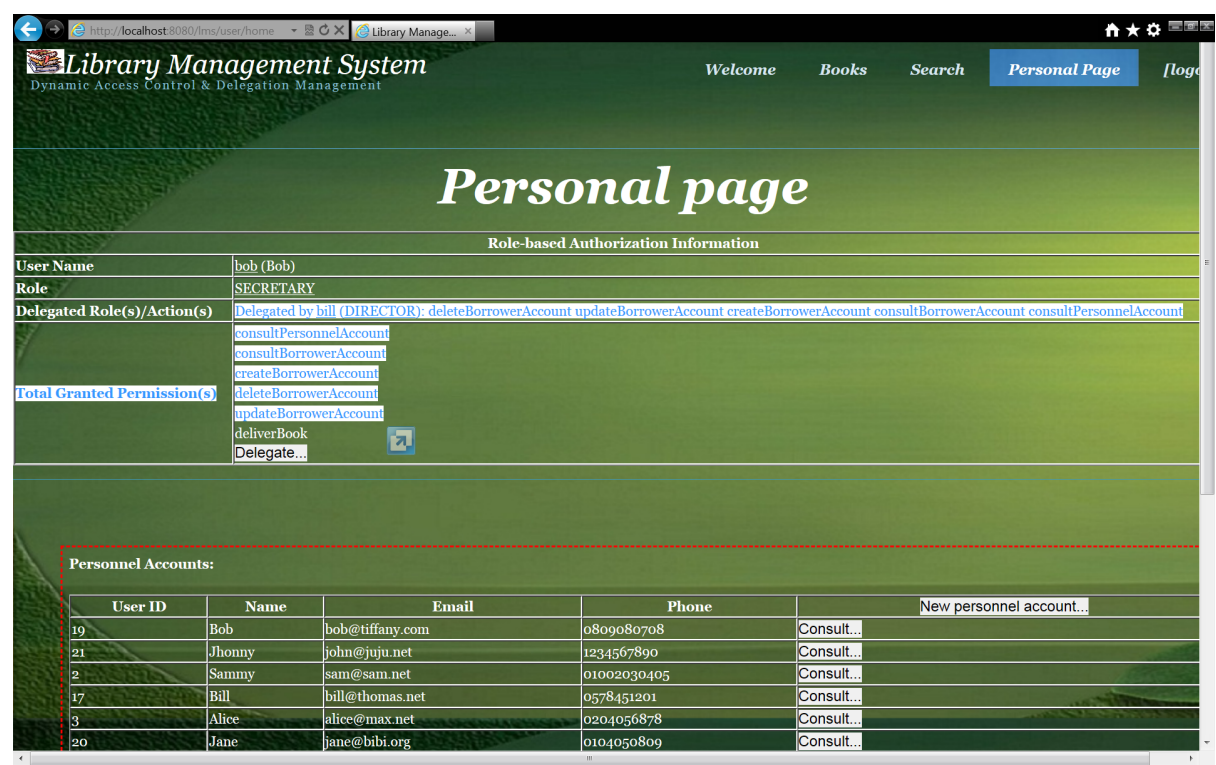

Fig. 10. Bob is delegated by Bill 5 permissions, e.g. consult personnel account

consultPersonnelAccount of the User proxy component Bob, via corresponding Role and Resource proxy components, to the real method consultPersonnelAccount in the business logic.

\subsection{Kevoree as the target Adaptive Execution Platform}

In case we use Kevoree as the target execution platform, all components (business logic components and proxy components) are implemented as Kevoree component instances [13]. The adaptation process is driven by a generated reconfiguration Kevoree script. The Kevoree script orchestrates the adaptation process of the running system by adding, removing component instances, binding the service ports between proxy components. An example of the configuration of proxy components (the 3-layer architecture) is shown in Figure 7. In order to explain how the proxy components are implemented, let's take a look at the Director Role proxy component. This Role proxy component is representative as it is between the User layer and the Resource layer (Figure 7). It can be seen that this Director Role proxy component provides the ports (services) to the User proxy components and also requires the ports (services) of the Resource proxy components. The code snippet in Listing 1.3 shows the ports required and provided by the Director Role proxy component. The required ports are bound to the corresponding ports provided by the BorrowerAccountResource proxy component and the PersonnelAccountResource proxy component. The provided ports are to be bound by the ports required by the corresponding User proxy components. 
Once the corresponding User proxy component calls the service provided by the port "deleteBorrowerAccountIn" (line 8, Listing 1.3), the method deleteBorrowerAccount (line 3, Listing 1.4) in the Director Role proxy component is executed that in turn calls the service provided by the port "deleteBorrowerAccountOut" (line 5, Listing 1.4. In fact this port is provided by the BorrowerAccountResource proxy component that finally calls to the corresponding method of deleteBorrowerAccount in the business logic code.

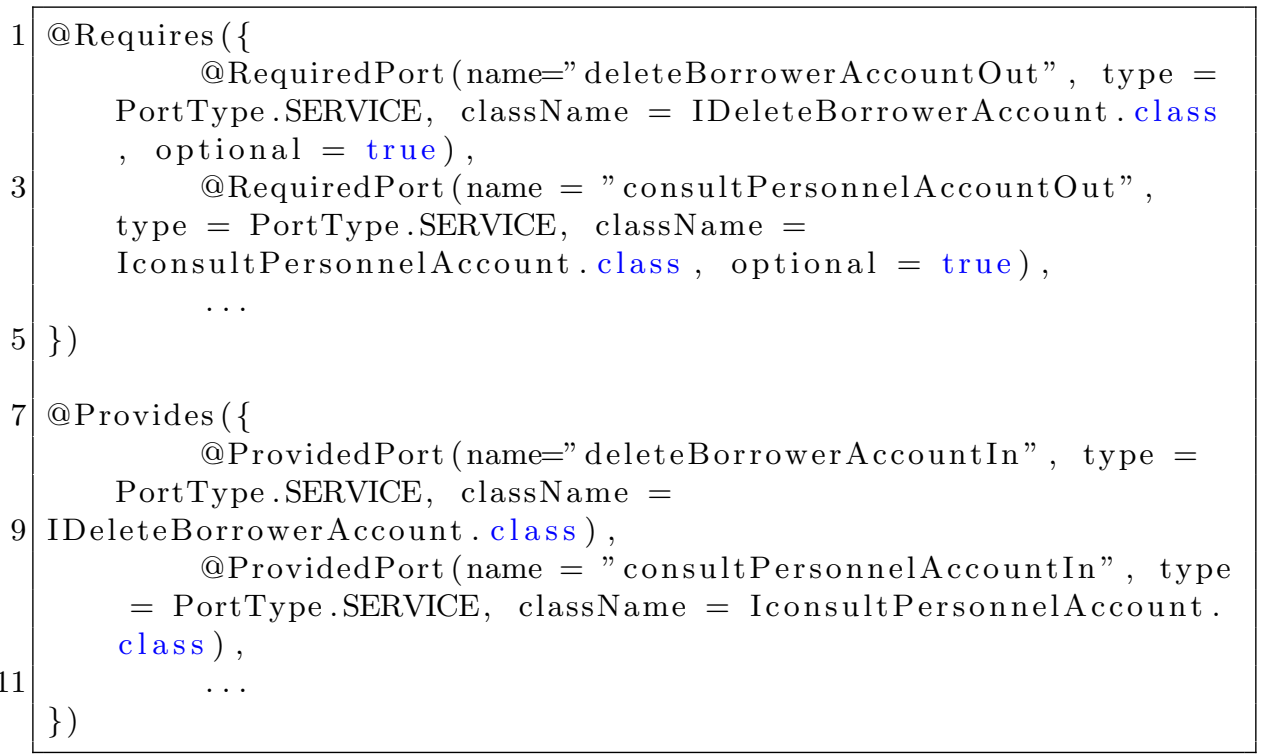

Listing 1.3. The ports required and provided by the Director Role proxy component

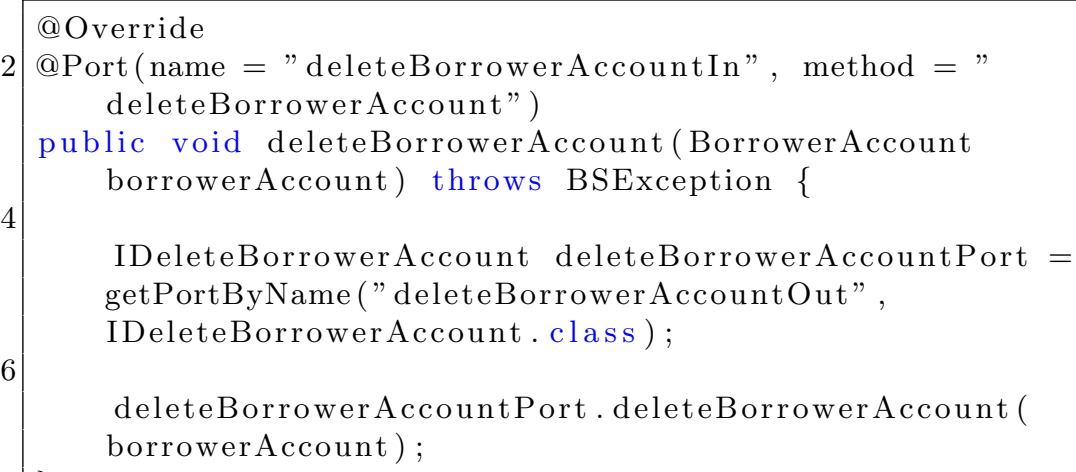

Listing 1.4. Redirecting the method call in the Director Role proxy component in Kevoree

There are three main advantages of using Kevoree over OSGi as the execution platform. Firstly, all we need to provide for the platform is the Kevoree reconfiguration script saying how to adapt the system. The Kevoree execution 
Table 3. Performance of weaving Security Policies using Kermeta and ATL

\begin{tabular}{|l|r|r|r|r|}
\hline & \# Rules & Kermeta 1.4.1 & Kermeta 2.0.6 & ATL 3.2.1 \\
\hline LMS & 27 & $4 \mathrm{~s}$ & $1.836 \mathrm{~s}$ & $0.048 \mathrm{~s}$ \\
\hline VMS & 44 & $7 \mathrm{~s}$ & $2.161 \mathrm{~s}$ & $0.055 \mathrm{~s}$ \\
\hline ASMS & 97 & $18 \mathrm{~s}$ & $2.834 \mathrm{~s}$ & $0.140 \mathrm{~s}$ \\
\hline
\end{tabular}

platform takes care of the necessary adaptation order for the running system according to changes. In case of using OSGi, we have to take care of the adaptation order manually. Secondly, the model@runtime environment of Kevoree makes it easier for implementing our model driven framework. In Kevoree, we can use the Kevoree framework itself to manage the security policy models. Thirdly, the way of declaring ports and bindings in Kevoree are very close to the concepts of ports and bindings described in our 3-layer architecture (Figure 7). This makes it very convenient to implement the running systems in Kevoree.

\subsection{Evaluation and Discussion}

There are two kinds of response time we would like to measure in our case studies: the authorization mechanism and the dynamic adaptation according to changing security policies. The experiments were performed on Intel Core i7 CPU 2.20 GHz with 2.91 GB usable RAM running on Windows 7. The number of security rules defined for each system in our experiments is indicated in Table 2. Because all our access control and delegation rules are transformed to proxy components reflecting our security policy, response times to an access request only depends on method calls between these proxy components and business components (Figure 7). Unsurprisingly, response time to every resource access is a constant, only about 1 millisecond, because the access is already possible or not by construction. In other words, our 3-layered architecture reflecting security policy enables very quick response, independently from the number of access control and delegation rules.

For experimenting with performance of adapting the running system, we have implemented the model transformation/composition rules using not only Kermeta but also ATL. Regarding the adaptation process, Table 3 shows results of each case study for performing the model transformations of security policies mentioned in Table 2, using Kermeta 1.4.1, Kermeta 2.0.6, and ATL 3.2.1 correspondingly. Note that these model-to-model transformations are generic, platform-independent w.r.t the implementation platform of the running system. Thus, the same model-to-model transformations are used in both cases of implementation platform, i.e. OSGi and Kevoree. At first, we used Kermeta 1.4.1 to implement our model transformations. However, the performance of using Kermeta 1.4.1 shown in Table 3 was disappointing. It took more than 18 seconds to weave 97 security rules in case of the ASMS. To know if this performance problem is inherently linked to our approach or simply linked to the use of Kermeta 1.4.1, we decided to also implement our model transformations using ATL 3.2.1. 
Our experiments show that the implementation using ATL 3.2.1 is much more efficient. We can conclude that the initial performance issue was due to Kermeta 1.4.1. Then, we have tried to use Kermeta 2.0.6 that is the latest version of Kermeta at this moment, compiled to byte code, which means much better performances. As can be seen from Table 3, the results of using Kermeta 2.0.6 are much better compared to using Kermeta 1.4.1.

Note that the transformation, code generation and compilation are performed "offline" meaning that the running system is not yet adapted. The actual adaptation happens when the newly compiled proxy components are integrated into the running system to replace the current proxy components. This actual adaptation process takes only some milliseconds by using the low-level APIs to reconfigure a system at runtime provided by the modern adaptive execution platforms, i.e. OSGi [33] and Kevoree [13]. Right after the new proxy components are up and running, the new security policy is really enforced in the running system.

\section{Related Work}

There is substantial work related to delegation as an extension of existing access control models. Most researchers focused on proposing models solely relying on the RBAC formalism [32], which is not expressive enough to deal with all delegation requirements. Therefore, some other researchers extended the RBAC model by adding new components, such as new types of roles, permissions and relationships $[2,34,1,9,27]$. In [5], the authors proposed yet another delegation approach for role-based access control (more precisely for ORBAC model) which is more flexible and comprehensive. However, no related work has provided a modeldriven approach for both specifying and dynamically enforcing access control policies with various delegation requirements. Compared to [24], we extend the model-based dynamic adaptation approach of [24] with some key improvements. More specifically, we propose a new DsmL for delegation management, but also new composition rules to weave delegation in a RBAC-based access control policy. In addition, we present a new way (by generating proxy) to implement the adaptation of the security-enforced architecture of the system. Indeed, we provide an extensive support for delegation as well as co-evolution of security policy and security-critical system. That means our approach makes it possible to deeply modify the security policy (e.g. according to evolution of the security-critical system) and dynamically adapt the running system, which is often infeasible using the other approaches mentioned above.

In addition, several researchers proposed new flexible access control models that may not include delegation, but allow a flexible and easy to update policy. For instance, Bertino et al. [6] proposed a new access control model that allows expressing flexible policies that can be easily modified and updated by users to be adapted to specific contexts. The advantage of their model resides in the ability to change the access control rules by granting or revoking the access based on specific exceptions. Their model provides a wide range of interesting features that increase the flexibility of the access control policy. It allows advanced ad- 
ministrative functions for regulating the specification of access controls rules. More importantly, their model supports delegation, enabling users to temporarily grant other users some of their permissions. Furthermore, Bertolissi et al. proposed DeBAC [7] a new access control model based on the notion of event that allows the policy to be adapted to distributed and changing environments. Their model is represented as a term rewriting system [3], which allows specifying changing and dynamic access control policies. This enables having a dynamic policy that is easy to change and update.

As far as we know, no previous work tackled the issue of enforcing adaptive delegation. Some previous approaches were proposed to help modeling more general access control formalisms using UML diagrams (focusing on models like RBAC or MAC). RBAC was modeled using a dedicated UML diagram template [17], while Doan et al. proposed a methodology [11] to incorporate MAC in UML diagrams during the design process. All these approaches allow access control formalisms to be expressed during the design. They do not provide a specific framework to enable adaptive delegation at runtime. Concerning the approaches related to applying MDE for security, we can cite UMLSEC [15], which is an extension of UML that allows security properties to be expressed in UML diagrams. In addition, Lodderstedt et al. [20] propose SECUREUML which provides a methodology for generating security components from specific models. The approach proposes a security modeling language to define the access control model. The resulting security model is combined with the UML business model in order to automatically produce the access control infrastructure. More precisely, they use the Meta-Object facility to create a new modeling language to define RBAC policies (extended to include constraints on rules). They apply their technique in different examples of distributed system architectures including Enterprise Java Beans and Microsoft Enterprise Services for .NET. Their approach provides a tool for specifying the access control rules along with the model-driven development process and then automatically exporting these rules to generate the access control infrastructure. However, they do not directly support delegation. Delegation rules should be taken into account early and the whole system should be generated again to enforce the new rules. Our approach enables supporting directly the delegation rules and dynamically enforcing them by reconfiguring the system at runtime.

\section{Conclusion and Future Work}

In this paper, we have proposed an extensive Model-Driven Security approach for adaptive delegation in access control management. By giving a formalization of access control and delegation mechanisms, we introduced various advanced delegation features that would provide secure, flexible, and efficient access control management. It has been shown that these advanced delegation features can be specified using our delegation DsmL. Our DsmL supports complex delegation characteristics like temporary, recurrence delegation, transfer delegation, multiple and multi-step delegation, etc. We have also shown that revocation can be 
dealt with in a simple manner. Another main contribution of this paper is our adaptive delegation enforcement in which delegation is considered as a "metalevel" mechanism that impacts the access control rules. A complete model-driven framework has been proposed to enable dynamic enforcement of delegation and access control policies that allows the automatic configuration of the system according to the changes in delegation/access control rules. Moreover, our framework also enables an adaptation strategy that better supports co-evolution of security policy and business logic of the system. The model-driven framework proposed in this paper can be applied for securing (distributed) systems running on different adaptive execution platform like OSGi (Equinox), or an optimized models@runtime framework such as Kevoree. Our approach has been validated via three different case studies with consideration of performance and extensibility issues.

In this paper, we only focus on the delegation of rights, further work will also be dedicated to the delegation of obligations and the support for usage control [31]. Usage control is called the next generation of access control with more flexible access management mechanisms that we would adopt our current approach for. We have not dealt with this idea yet in this paper, but keep it for our future work. Moreover, revocation mechanism in our current approach has not been completely taken into account, i.e. without options of strong/weak revocation. Besides, in order to complete the framework, we also propose an approach for testing delegation policy enforcement. In this direction, we continue working on the extension of testing delegation policy enforcement via mutation analysis [29].

Acknowledgments. We would like to thank the anonymous referees for their comments and suggestions. This work is supported by the Fonds National de la Recherche (FNR), Luxembourg, under the MITER project C10/IS/783852.

\section{References}

1. G.-J. Ahn, B. Mohan, and S.-P. Hong. Towards secure information sharing using role-based delegation. J. Netw. Comput. Appl., 30(1):42-59, 2007.

2. E. Barka and R. Sandhu. Role-based delegation model/hierarchical roles (RBDM1). In Proceedings of the 20th Annual Computer Security Applications Conference, ACSAC '04, pages 396-404. IEEE Computer Society, 2004.

3. S. Barker and M. Fernández. Term rewriting for access control. In DBSec, pages 179-193, 2006.

4. D. Basin, J. Doser, and T. Lodderstedt. Model Driven Security: From UML models to access control infrastructures. ACM Trans. Softw. Eng. Methodol., 15(1):39-91, Jan. 2006.

5. M. Ben-Ghorbel-Talbi, F. Cuppens, N. Cuppens-Boulahia, and A. Bouhoula. A delegation model for extended RBAC. Int. J. Inf. Secur., 9(3):209-236, June 2010.

6. E. Bertino, S. Jajodia, and P. Samarati. A flexible authorization mechanism for relational data management systems. ACM Trans. Inf. Syst., 17(2):101-140, 1999. 
7. C. Bertolissi, M. Fernández, and S. Barker. Dynamic event-based access control as term rewriting. In DBSec, pages 195-210, 2007.

8. E. Bruneton, T. Coupaye, M. Leclercq, V. Quéma, and J. Stefani. The Fractal component model and its support in Java. Software Practice and Experience, Special Issue on Experiences with Auto-adaptive and Reconfigurable Systems, 36(1112):1257-1284, 2006.

9. J. Crampton and H. Khambhammettu. Delegation in role-based access control. International Journal of Information Security, 7(2):123-136, 2008.

10. F. Cuppens and N. Cuppens-Boulahia. Modeling contextual security policies. International Journal of Information Security, 7(4):285-305, Nov. 2007.

11. T. Doan, S. Demurjian, T. C. Ting, and A. Ketterl. MAC and UML for secure software design. In FMSE '04: Proceedings of the 2004 ACM workshop on Formal methods in security engineering, pages 75-85. ACM, 2004.

12. D. F. Ferraiolo, R. Sandhu, S. Gavrila, D. R. Kuhn, and R. Chandramouli. Proposed NIST standard for role-based access control. ACM Trans. Inf. Syst. Secur., 4(3):224-274, Aug. 2001.

13. F. Fouquet, G. Nain, B. Morin, E. Daubert, O. Barais, N. Plouzeau, and J.-M. Jzquel. An Eclipse Modelling Framework Alternative to meet the Models@Runtime requirements. In R. France, J. Kazmeier, R. Breu, and C. Atkinson, editors, Model Driven Engineering Languages and Systems, volume 7590 of Lecture Notes in Computer Science, pages 87-101. Springer Berlin Heidelberg, 2012.

14. S. Jajodia, P. Samarati, M. L. Sapino, and V. S. Subrahmanian. Flexible support for multiple access control policies. ACM Trans. Database Syst., 26(2):214-260, 2001.

15. J. Jürjens. UMLsec: Extending UML for secure systems development. In UML'02: 5th International Conference on The UML, pages 412-425. Springer-Verlag, 2002.

16. A. Kalam, R. Baida, P. Balbiani, S. Benferhat, F. Cuppens, Y. Deswarte, A. Miege, C. Saurel, and G. Trouessin. Organization based access control. In Policies for Distributed Systems and Networks, 2003. Proceedings. POLICY 2003. IEEE 4th International Workshop on, pages 120-131, 2003.

17. D.-K. Kim, I. Ray, R. B. France, and N. Li. Modeling role-based access control using parameterized UML models. In FASE, pages 180-193, 2004.

18. B. Klatt. Xpand: A closer look at the model2text transformation language. Language, (10/16/2008), 2007.

19. B. W. Lampson. Protection. SIGOPS Oper. Syst. Rev., 8(1):18-24, Jan. 1974.

20. T. Lodderstedt, D. Basin, and J. Doser. SecureUML: A UML-based modeling language for Model-Driven Security. In UML'02: 5th International Conference on The UML, pages 426-441. Springer-Verlag, 2002.

21. B. Morin, O. Barais, J.-M. Jézéquel, F. Fleurey, and A. Solberg. Models@ Run.time to support dynamic adaptation. Computer, 42(10):44-51, Oct. 2009.

22. B. Morin, O. Barais, G. Nain, and J.-M. Jézéquel. Taming dynamically adaptive systems with Models and Aspects. In ICSE'09: 31st International Conference on Software Engineering, May 2009.

23. B. Morin, F. Fleurey, N. Bencomo, J.-M. Jézéquel, A. Solberg, V. Dehlen, and G. Blair. An Aspect-Oriented and Model-Driven approach for managing dynamic variability. In Proceedings of the 11th international conference on Model Driven Engineering Languages and Systems, MoDELS '08, pages 782-796. Springer-Verlag, 2008.

24. B. Morin, T. Mouelhi, F. Fleurey, Y. Le Traon, O. Barais, and J.-M. Jézéquel. Security-driven model-based dynamic adaptation. In Proceedings of 
the IEEE/ACM international conference on Automated software engineering, ASE '10, pages 205-214. ACM, 2010.

25. T. Mouelhi, Y. Le Traon, and B. Baudry. Transforming and selecting functional test cases for security policy testing. In Proceedings of the 2009 International Conference on Software Testing Verification and Validation, ICST '09, pages 171180. IEEE Computer Society, 2009.

26. P.-A. Muller, F. Fleurey, and J.-M. Jézéquel. Weaving executability into objectoriented meta-languages. In International Conference on Model Driven Engineering Languages and Systems (MoDELS), LNCS 3713, pages 264-278. Springer, 2005.

27. S. Na and S. Cheon. Role delegation in role-based access control. In Proceedings of the fifth ACM workshop on Role-based access control, RBAC '00, pages 39-44. ACM, 2000.

28. P. H. Nguyen, G. Nain, J. Klein, T. Mouelhi, and Y. Le Traon. Model-driven adaptive delegation. In Proceedings of the 12th annual International Conference on Aspect-Oriented Software Development, Modularity:AOSD '13, pages 61-72. ACM, 2013.

29. P. H. Nguyen, M. Papadakis, and I. Rubab. Testing delegation policy enforcement via mutation analsysis. In Proceedings of the Workshop on Mutation Testing @ the Sixth IEEE International Conference on Software Testing, ICST'13, pages 61-72. IEEE, 2013.

30. D. Rubio. Pro Spring dynamic modules for OSGi service platforms. 2009.

31. R. Sandhu and J. Park. Usage Control: A vision for next generation access control. V. Gorodetsky et al. (Eds.): MMM-ACNS 2003, LNCS 2776, 1:17-31, 2003.

32. R. S. Sandhu, E. J. Coyne, H. L. Feinstein, and C. E. Youman. Role-based access control models. Computer, 29(2):38-47, Feb. 1996.

33. O. The OSGi Alliance. OSGi service platform core specification, release 4.1. 2007.

34. X. Zhang, S. Oh, and R. Sandhu. PBDM: a flexible delegation model in RBAC. In Proceedings of the eighth ACM symposium on Access control models and technologies, SACMAT '03, pages 149-157. ACM, 2003. 